Journal of Circuits, Systems, and Computers

Vol. 18, No. 3 (2009) 465-486

(C) World Scientific Publishing Company

\title{
EXACT MINIMIZATION OF AND-EXOR EXPRESSIONS OF PRACTICAL BENCHMARK FUNCTIONS
}

\author{
TAKASHI HIRAYAMA* and YASUAKI NISHITANI ${ }^{\dagger}$ \\ Department of Computer and Information Sciences, \\ Iwate University, 4-3-5 Ueda, \\ Morioka, Iwate 020-8551, Japan \\ *hirayama@kono.cis.iwate-u.ac.jp \\ †nisitani@kono.cis.iwate-u.ac.jp
}

Revised 13 November 2008

\begin{abstract}
We propose faster-computing methods for the minimization algorithm of AND-EXOR expressions, or Exclusive-or Sum-Of-Products expressions (ESOPs), and obtain the exact minimum ESOPs of benchmark functions. These methods improve the search procedure for ESOPs, which is the most time-consuming part of the original algorithm. For faster computation, the search space for ESOPs is reduced by checking the upper and lower bounds on the size of ESOPs. Experimental results to demonstrate the effectiveness of these methods are presented. The exact minimum ESOPs of many practical benchmark functions have been revealed by this improved algorithm.
\end{abstract}

Keywords: AND-EXOR two-level circuit; AND-EXOR expression; exclusive-or sum-ofproducts expression; logic minimization algorithm.

\section{Introduction}

Logic circuits including exclusive-or (EXOR) gates have some advantages over traditional circuits with only AND and OR gates. EXOR-based realization can improve the testability ${ }^{1,2,3,4}$ and often reduces the circuit area. ${ }^{5,6,7,8}$ For arithmetic functions, error-correcting functions, and telecommunication functions, AND-EXOR circuits are smaller than AND-OR ones. ${ }^{9}$ AND-EXOR logic expressions, which correspond to AND-EXOR two-level circuits, have been studied as the fundamentals of the EXOR-based realization. Current applications of AND-EXOR expressions are logic synthesis of new type of circuits such as reversible logic circuits ${ }^{10}$ and quantum circuits. ${ }^{11}$

There are several classes of AND-EXOR expressions ${ }^{12,13}$ such as positivepolarity Reed-Muller expressions (PPRMs), fixed-polarity Reed-Muller expressions (FPRMs), double-fixed-polarity Reed-Muller expressions (DFPRMs), and exclusive-or sum-of-products expressions (ESOPs). ESOPs are the expressions such that arbitrary product terms are combined by EXORs. Among the classes of ANDEXOR expressions, ESOPs are the most general expressions and require the fewest 
product terms to represent logic functions. The number of product terms of an ESOP $F$ is called the size of $F$. Among all ESOPs that represent a logic function $f$, those with the minimum size are called the exact minimum ESOPs of $f$, the size of which is called the size of $f$ for short.

In this paper, minimization means to obtain the exact minimum ESOP of $f$ and simplification means to reduce the number of product terms in ESOPs without guaranteeing minimality. In general, minimization is much more time-consuming than simplification. The exact minimum results, however, can be used to evaluate the performance of the simplification algorithms, or may be helpful in theoretical analysis of EXOR-based synthesis such as reversible logic and/or quantum circuits. As an application to the reversible logic synthesis, the exact minimization of a general class of ESOPs, called exclusive-or sum of complex terms (ESCTs), has been studied ${ }^{34}$.

A lot of algorithms for simplifying ESOPs by applying heuristic rewriting rules have been proposed ${ }^{14,15,16,17,19}$; Sasao's, ${ }^{18}$ Mishchenko-Perkowski's,${ }^{20}$ and StergiouVoudouris-Papakonstantinou's ${ }^{38}$ algorithms are known to be especially efficient. However, these algorithms do not guarantee the minimality of the resulting ESOPs. On the other hand, studies of algorithms for minimizing ESOPs are fewer; ${ }^{21,22,23,37}$ so far, no efficient minimization algorithms for ESOPs are known. It is considered difficult to minimize ESOPs. In practice, previous minimization algorithms are applicable only to small functions, e.g. functions whose size is less than eight ${ }^{23}$ or functions with at most six variables. ${ }^{24,37}$

In this paper, we present some acceleration methods for a minimization algorithm of ESOPs. By adding these methods to the base algorithm, ${ }^{24}$ a more powerful algorithm can be constructed. It computes minimum ESOPs of several benchmark functions. In Section 2, we give some definitions and refer to the basic algorithms for minimization. The efficient methods for time and memory are discussed in Section 3. Section 4 extends our algorithms to minimization of multiple-output functions. Section 5 shows the minimum ESOPs for benchmark functions obtained by our algorithms as experimental results. The conclusion is given in Section 6 .

\section{Preliminaries}

In this section, we refer to the basic algorithm for minimizing ESOPs.

Definition 1. The size of an ESOP $F$ is the number of product terms of $F$, denoted by $\tau(F)$. Among all ESOPs that represent a logic function $f$, those with the minimum size are called minimum ESOPs of $f$. The size of a minimum ESOP of $f$ is denoted by $\tau(f)$.

Example 1. The following logic expressions $F_{1}, F_{2}$, and $F_{3}$ are ESOPs that represent the same logic function $f$ given in Table 1.

$$
\begin{aligned}
F_{1}= & \bar{x}_{4} \bar{x}_{3} \bar{x}_{2} \bar{x}_{1} \oplus \bar{x}_{4} \bar{x}_{3} \bar{x}_{2} x_{1} \oplus \bar{x}_{4} \bar{x}_{3} x_{2} \bar{x}_{1} \oplus \bar{x}_{4} \bar{x}_{3} x_{2} x_{1} \oplus \bar{x}_{4} x_{3} \bar{x}_{2} x_{1} \oplus \bar{x}_{4} x_{3} x_{2} \bar{x}_{1} \oplus \\
& x_{4} \bar{x}_{3} \bar{x}_{2} \bar{x}_{1} \oplus x_{4} x_{3} \bar{x}_{2} \bar{x}_{1} \oplus x_{4} x_{3} \bar{x}_{2} x_{1} \oplus x_{4} x_{3} x_{2} \bar{x}_{1} \oplus x_{4} x_{3} x_{2} x_{1}
\end{aligned}
$$


Table 1. Truth table of an example function $f$

\begin{tabular}{cccc|c}
\hline$x_{4}$ & $x_{3}$ & $x_{2}$ & $x_{1}$ & $f$ \\
\hline 0 & 0 & 0 & 0 & 1 \\
0 & 0 & 0 & 1 & 1 \\
0 & 0 & 1 & 0 & 1 \\
0 & 0 & 1 & 1 & 1 \\
0 & 1 & 0 & 0 & 0 \\
0 & 1 & 0 & 1 & 1 \\
0 & 1 & 1 & 0 & 1 \\
0 & 1 & 1 & 1 & 0 \\
1 & 0 & 0 & 0 & 1 \\
1 & 0 & 0 & 1 & 0 \\
1 & 0 & 1 & 0 & 0 \\
1 & 0 & 1 & 1 & 0 \\
1 & 1 & 0 & 0 & 1 \\
1 & 1 & 0 & 1 & 1 \\
1 & 1 & 1 & 0 & 1 \\
1 & 1 & 1 & 1 & 1 \\
\hline
\end{tabular}

$$
\begin{aligned}
& F_{2}=x_{4} x_{3} x_{2} x_{1} \oplus x_{4} x_{3} \oplus x_{4} x_{2} x_{1} \oplus x_{4} x_{2} \oplus x_{4} x_{1} \oplus x_{3} x_{2} \oplus x_{3} x_{1} \oplus x_{3} \oplus 1 \\
& F_{3}=\bar{x}_{4} x_{3} \bar{x}_{2} \bar{x}_{1} \oplus \bar{x}_{4} x_{3} \bar{x}_{2} \oplus \bar{x}_{4} x_{3} \bar{x}_{1} \oplus \bar{x}_{4} \bar{x}_{2} \bar{x}_{1} \oplus \bar{x}_{4} \oplus x_{3} \bar{x}_{2} \bar{x}_{1} \oplus x_{3} \oplus \bar{x}_{2} \bar{x}_{1}
\end{aligned}
$$

The size of the above ESOPs are $\tau\left(F_{1}\right)=11, \tau\left(F_{2}\right)=9$, and $\tau\left(F_{3}\right)=8$. These are ESOPs of $f$ but not minimum ones in size. The minimum ESOP of $f$ is $F_{4}=$ $\bar{x}_{4} x_{3} \bar{x}_{1} \oplus \bar{x}_{4} x_{2} \oplus x_{4} \bar{x}_{3} \bar{x}_{2} x_{1} \oplus \bar{x}_{3} x_{2} \oplus 1$, whose size is $\tau\left(F_{4}\right)=5$. Thus $\tau(f)=5$.

Definition 2. For an $n$-variable function $f$ and a variable $x$, the subfunctions of $f$ with $x=0$ and $x=1$ are denoted by $f_{x:\{0\}}$ and $f_{x:\{1\}}$, respectively. Furthermore $f_{x:\{0,1\}}$ and $f_{x:\{\}}$ are defined as $f_{x:\{0\}} \oplus f_{x:\{1\}}$ and the logical zero function 0 , respectively. In general, $f_{x:(I \oplus J)}(I, J \subseteq\{0,1\}, I \oplus J=(I \cup J)-(I \cap J))$ is defined as $f_{x: I} \oplus f_{x: J}$.

Example 2. Table 2 shows the subfunctions $f_{x_{4}:\{0\}}, f_{x_{4}:\{1\}}$, and $f_{x_{4}:\{0,1\}}$ of $f$ given in Example 1. These subfunctions may be written as $f_{x_{4}:\{0,1\}}=$ $f_{x_{4}:(\{0\} \oplus\{1\})}=f_{x_{4}:\{0\}} \oplus f_{x_{4}:\{1\}}, f_{x_{4}:\{0\}}=f_{x_{4}:(\{1\} \oplus\{0,1\})}=f_{x_{4}:\{1\}} \oplus f_{x_{4}:\{0,1\}}$, and $f_{x_{4}:\{1\}}=f_{x_{4}:(\{0\} \oplus\{0,1\})}=f_{x_{4}:\{0\}} \oplus f_{x_{4}:\{0,1\}}$.

Table 2. Truth tables of subfunctions of $f$

\begin{tabular}{ccc|c}
\hline$x_{3}$ & $x_{2}$ & $x_{1}$ & $f_{x_{4}:\{0\}}$ \\
\hline 0 & 0 & 0 & 1 \\
0 & 0 & 1 & 1 \\
0 & 1 & 0 & 1 \\
0 & 1 & 1 & 1 \\
1 & 0 & 0 & 0 \\
1 & 0 & 1 & 1 \\
1 & 1 & 0 & 1 \\
1 & 1 & 1 & 0 \\
\hline
\end{tabular}

\begin{tabular}{ccc|c}
\hline$x_{3}$ & $x_{2}$ & $x_{1}$ & $f_{x_{4}:\{1\}}$ \\
\hline 0 & 0 & 0 & 1 \\
0 & 0 & 1 & 0 \\
0 & 1 & 0 & 0 \\
0 & 1 & 1 & 0 \\
1 & 0 & 0 & 1 \\
1 & 0 & 1 & 1 \\
1 & 1 & 0 & 1 \\
1 & 1 & 1 & 1 \\
\hline
\end{tabular}

\begin{tabular}{ccc|c}
\hline$x_{3}$ & $x_{2}$ & $x_{1}$ & $f_{x_{4}:\{0,1\}}$ \\
\hline 0 & 0 & 0 & 0 \\
0 & 0 & 1 & 1 \\
0 & 1 & 0 & 1 \\
0 & 1 & 1 & 1 \\
1 & 0 & 0 & 1 \\
1 & 0 & 1 & 0 \\
1 & 1 & 0 & 0 \\
1 & 1 & 1 & 1 \\
\hline
\end{tabular}


With subfunctions in Definition 2, an arbitrary function $f$ can be expanded as follows, ${ }^{13}$ where $I \subseteq\{0,1\}$.

$$
f=x f_{x:(I \oplus\{0\})} \oplus \bar{x} f_{x:(I \oplus\{1\})} \oplus f_{x:(I \oplus\{0,1\})}
$$

The expansion with $I=\{0,1\}$ is known as the Shannon expansion, and the expansions with $I=\{1\}$ and $I=\{0\}$ are known as the positive Davio and the negative Davio expansions, respectively. With an arbitrary $(n-1)$-variable function $g$, a more general expansion holds:

$$
f=x\left(f_{x:(I \oplus\{0\})} \oplus g\right) \oplus \bar{x}\left(f_{x:(I \oplus\{1\})} \oplus g\right) \oplus\left(f_{x:(I \oplus\{0,1\})} \oplus g\right),
$$

since $(x \oplus \bar{x} \oplus 1) g=0$. Equation (1) is a special case of Eq. (2) with $g=0$.

Definition 3. Let $f$ and $g$ be functions with $n$ variables and $(n-1)$ variables, respectively. $T_{x: I}(f, g)(I \subseteq\{0,1\})$ and $T(f, g)$ are defined as follows.

$$
\begin{aligned}
T_{x: I}(f, g) & =\tau\left(f_{x:(I \oplus\{0\})} \oplus g\right)+\tau\left(f_{x:(I \oplus\{1\})} \oplus g\right)+\tau\left(f_{x:(I \oplus\{0,1\})} \oplus g\right) \\
T(f, g) & =\min \left\{T_{x:\{0\}}(f, g), T_{x:\{1\}}(f, g), T_{x:\{0,1\}}(f, g)\right\}
\end{aligned}
$$

The following theorem is the basic principle of minimization. ${ }^{25}$

Theorem 1. (Minimization Theorem) Let $\mathcal{F}^{n-1}$ be the set of all $(n-1)$ variable functions. For an arbitrary $n$-variable function $f$, the following equation holds.

$$
\tau(f)=\min \left\{T(f, g) \mid g \in \mathcal{F}^{n-1}\right\}
$$

From Theorem 1, we can construct a simple minimization algorithm such that all $(n-1)$-variable functions $g \in \mathcal{F}^{n-1}$ are tested, which is shown as min-esop ${ }^{26}$ in Fig 1. In the algorithm, a minimum ESOP of an arbitrary $n$-variable function $f$ for $n \leq n^{\prime}$ and its number of products $\tau(f)$ are assumed to be known as the terminal condition of the recursive algorithm. For example, $n^{\prime}$ can be 5 since minimum ESOPs for the representative functions with five or less variables has been presented. ${ }^{27,28}$ In min-esop, a minimum ESOP of $f$ can be obtained together with $\tau(f)$, that is, a minimum ESOP and $\tau(f)$ can be obtained by the same algorithm. Although, in the rest of this paper, we focus on obtaining $\tau(f)$ to simplify the discussion, it includes obtaining a minimum ESOP on the analogy of Theorem 1 and min-esop.

In the literature ${ }^{24}$, we presented a faster algorithm for minimizing ESOPs, which is called min-tau (shown in Fig. 2). We refer to the algorithm briefly in the following. Algorithm min-tau tests $(n-1)$-variable functions $g$ such that $\tau(g) \leq k$, where $k$ is an upper bound on the size of $g^{*}$ such that $T\left(f, g^{*}\right)=\tau(f)$. The value of $k$ is obtained by " $k:=\min \{\lfloor(s-1) / 3\rfloor, s-1-\gamma(f)\}$." $\gamma(f)$ is defined as $\gamma(f)=$ $\max \left\{\tau\left(f_{x:\{0\}}\right), \tau\left(f_{x:\{1\}}\right), \tau\left(f_{x:\{0,1\}}\right)\right\}$ and $s$ represents the smallest result so far. The upper bound $k$ is based on the property that there exists $g^{*} \in \mathcal{G}$ such that $T\left(f, g^{*}\right)=$ 


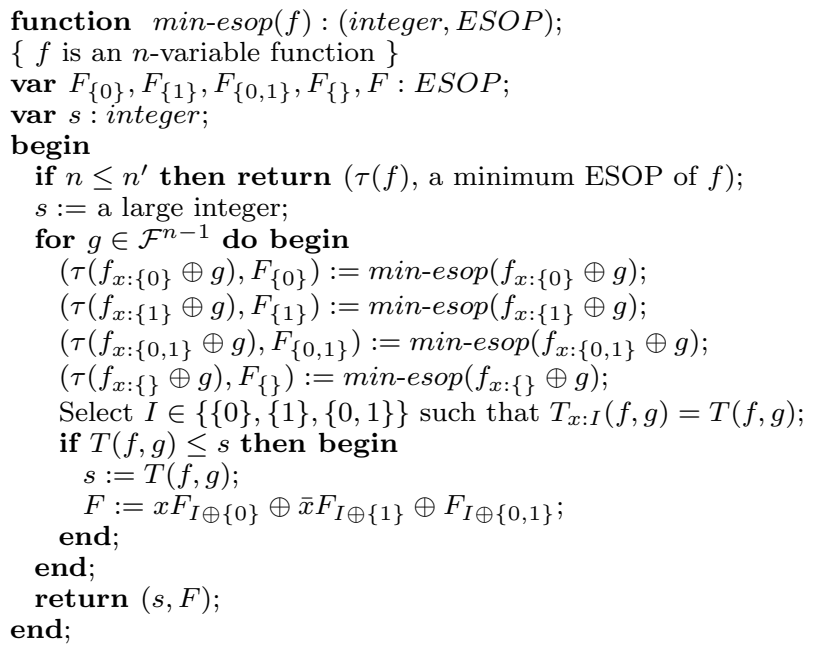

Fig. 1. min-esop: a simple minimization algorithm

$\tau(f)$, where $\mathcal{G}$ is the set of $g$ such that $\tau(g) \leq \tau(f) / 3$ and $\tau(g) \leq \tau(f)-\gamma(f)$. Here is some intuition for $\tau(g) \leq \tau(f) / 3$ and $\tau(g) \leq \tau(f)-\gamma(f)$. A minimum ESOP $F$ is written in the form $F=x G_{0} \oplus \bar{x} G_{1} \oplus G_{2}$. Function $g^{*}$ can be any of these $G_{i}$ and hence suffices to be the smallest of the three. Thus, the upper bound on the size of such $g^{*}$ is $\tau\left(g^{*}\right) \leq \tau(f) / 3$. For the minimum ESOP, $x G_{0} \oplus \bar{x} G_{1} \oplus G_{2}=x\left(f_{x:(I \oplus\{0\})} \oplus\right.$ $\left.g^{*}\right) \oplus \bar{x}\left(f_{x:(I \oplus\{1\})} \oplus g^{*}\right) \oplus\left(f_{x:(I \oplus\{0,1\})} \oplus g^{*}\right)$ holds for some $I$. The index may be $I=\{0,1\}$ for example. Then we have $\tau(f)=\tau\left(f_{x:\{1\}} \oplus g^{*}\right)+\tau\left(f_{x:\{0\}} \oplus g^{*}\right)+\tau\left(g^{*}\right)$. From this inequality and the property $\tau\left(f_{x:\{1\}} \oplus g^{*}\right)+\tau\left(f_{x:\{0\}} \oplus g^{*}\right) \geq \tau\left(f_{x:\{1\}} \oplus\right.$ $\left.g^{*} \oplus f_{x:\{0\}} \oplus g^{*}\right)=\tau\left(f_{x:\{0,1\}}\right)$, the inequality $\tau(f) \geq \tau\left(f_{x:\{0,1\}}\right)+\tau\left(g^{*}\right)$ holds. By transposing the terms, we have $\tau\left(g^{*}\right) \leq \tau(f)-\tau\left(f_{x:\{0,1\}}\right)$. Since the other cases of $I(I=\{0\}$ and $I=\{1\})$ are also checked in the algorithm, the inequality can be $\tau\left(g^{*}\right) \leq \tau(f)-\gamma(f)$.

The candidate functions for $g^{*}$ are generated dynamically as $g \oplus p$ in $S\left(g, \mathcal{P}, t_{f g}\right)$ from the arguments $g$ and $\mathcal{P}$. $\mathcal{P}^{n-1}$ used as the initial $\mathcal{P}$ is the set of all $(n-1)$ variable functions that can be represented by exactly one product. The variables $t_{f g}$ and $t_{f g p}$ are used to store the results of $T(f, g)$ and $T(f, g \oplus p)$, respectively. The simple minimization algorithm min-esop requires very long computation time since it tests all $g \in \mathcal{F}^{n-1}\left(\left|\mathcal{F}^{n-1}\right|=2^{2^{n-1}}\right)$. On the other hand, min-tau is much faster since it omits to test $g$ when $t_{f g}-(k-\tau(g)) \geq s$, where $(k-\tau(g))$ is an upper bound on the possible reduction of the size of $T(f, g)$. Holding the condition $t_{f g}-(k-\tau(g)) \geq s$ guarantees that further reduction of $T(f, g)$ cannot yield smaller results than the current smallest result $s$. This is why the procedure omits the computation in that case and returns. It has been proved that min-tau computes $\tau(f) .{ }^{24}$ The values of $T(f, 0)$ and $\gamma(f)$ to obtain the initial $k$ can be computed by applying the minimization algorithm min-tau recursively. Similarly, $\tau(g \oplus p)$ and 
$T(f, g \oplus p)$ in $S\left(g, \mathcal{P}, t_{f g}\right)$ are computed by applying min-tau recursively. Although the value of $\tau(g)$ is passed to the procedure $S\left(g, \mathcal{P}, t_{f g}\right)$ as its additional argument to avoid computing the same values in practical programming, the declaration of the argument is omitted in Fig. 2 for the reason of simplicity of the description.

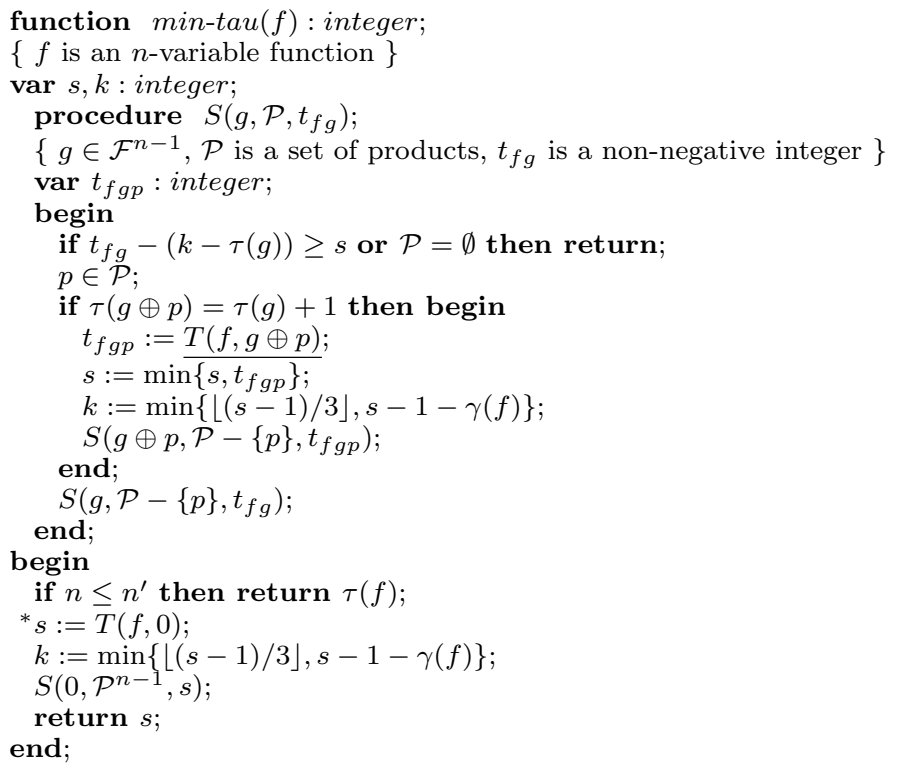

Fig. 2. min-tau: a minimization algorithm

\section{Faster Computation}

In this section, the acceleration based on min-tau is discussed. $T(f, g \oplus p)$ indicated by the underline in Fig. 2 is one of the most time-consuming part in this algorithm because the value is computed by applying min-tau recursively. We propose some methods to reduce the computation in $T(f, g \oplus p)$ by utilizing the information of neighborhoods of subfunctions: the lower bounds on the size of subfunctions in Section 3.1 and the upper bounds in Section 3.2.

\subsection{Breaking Conditions for $T(f, g \oplus p)$}

In Fig. 2, the procedure $S\left(g, \mathcal{P}, t_{f g}\right)$ computes $T(f, g \oplus p)$ as indicated by the underline. In this section, we discuss the acceleration in the case of

$$
T(f, g \oplus p) \geq s+k-\tau(g \oplus p) .
$$

Such a case happens frequently in the computation of min-tau since $T(f, g \oplus p)$ (a temporal solution) tends to be larger enough than $s$ (the smallest solution so far). 
If the inequality (3) holds, we have $T(f, g \oplus p) \geq s$ because of $k-\tau(g \oplus p) \geq 0$ from the property of min-tau. ${ }^{24}$ In this case, the result of " $t_{f g p}:=T(f, g \oplus p)$ " will not be utilized in the later computation. Specifically, from $t_{f g p}=T(f, g \oplus p) \geq s$, "s $:=$ $\min \left\{s, t_{f g p}\right\}$ " does not change the value of $s$, and hence the value of $k$ is unchanged. The succeeding recursive call $S\left(g \oplus p, \mathcal{P}-\{p\}, t_{f g p}\right)$ terminates immediately since the terminal condition of $S\left(g \oplus p, \mathcal{P}-\{p\}, t_{f g p}\right), t_{f g p}-(k-\tau(g \oplus p)) \geq s$, is satisfied by the inequality (3). Therefore, when the inequality (3) holds in advance or in the middle of the computation, the computation of $T(f, g \oplus p)$ can be broken off and any integer $t$ such that $t \geq s+k-\tau(g \oplus p)$ can be used instead of $T(f, g \oplus p)$. This idea is summarized below.

Property 1. In the procedure $S\left(g, \mathcal{P}, t_{f g}\right)$, replacement of $T(f, g \oplus p)$ by a function $T^{\prime}(f, g, p)$ does not change the return value of $S\left(g, \mathcal{P}, t_{f g}\right)$ if $T^{\prime}(f, g, p)$ has the following properties, where $l=s+k-\tau(g \oplus p)$.

(1) If $T(f, g \oplus p)<l, T^{\prime}(f, g, p)=T(f, g \oplus p)$.

(2) If $T(f, g \oplus p) \geq l, T^{\prime}(f, g, p) \geq l$.

From this idea, we modify $T(f, g \oplus p)$ to check the inequality (3) by using $\tau\left(f_{x:\{0\}} \oplus g\right), \tau\left(f_{x:\{1\}} \oplus g\right)$, and $\tau\left(f_{x:\{0,1\}} \oplus g\right)$. These values can be obtained at the computation of $T(f, g)$, which is done prior to $T(f, g \oplus p)$. From Definition 3, $T(f, g \oplus p)$ is computed from four values: $\tau\left(f_{x:\{0\}} \oplus g \oplus p\right), \tau\left(f_{x:\{1\}} \oplus g \oplus p\right)$, $\tau\left(f_{x:\{0,1\}} \oplus g \oplus p\right)$, and $\tau(g \oplus p)$. To discuss the breaking conditions of $T(f, g \oplus p)$, function $M$ with four arguments is defined.

Definition 4. $M\left(t_{0}, t_{1}, t_{2}, t_{3}\right)=\min \left\{t_{3}+t_{2}+t_{1}, t_{2}+t_{3}+t_{0}, t_{1}+t_{0}+t_{3}\right\}$

From Definitions 3 and $4, T(f, g \oplus p)=M\left(\tau\left(f_{x:\{0\}} \oplus g \oplus p\right), \tau\left(f_{x:\{1\}} \oplus g \oplus p\right)\right.$, $\left.\tau\left(f_{x:\{0,1\}} \oplus g \oplus p\right), \tau(g \oplus p)\right)$ holds. Since $p$ is a product, the inequalities

$$
\begin{gathered}
\tau\left(f_{x:\{0\}} \oplus g \oplus p\right) \geq \tau\left(f_{x:\{0\}} \oplus g\right)-1 \\
\tau\left(f_{x:\{1\}} \oplus g \oplus p\right) \geq \tau\left(f_{x:\{1\}} \oplus g\right)-1 \\
\tau\left(f_{x:\{0,1\}} \oplus g \oplus p\right) \geq \tau\left(f_{x:\{0,1\}} \oplus g\right)-1
\end{gathered}
$$

hold. By using $\tau\left(f_{x:\{0\}} \oplus g\right)-1, \tau\left(f_{x:\{1\}} \oplus g\right)-1$, and $\tau\left(f_{x:\{0,1\}} \oplus g\right)-1$, lower bounds of $T(f, g \oplus p)$ are written by $M$.

$$
\begin{aligned}
& T(f, g \oplus p) \\
& =M\left(\tau\left(f_{x:\{0\}} \oplus g \oplus p\right), \tau\left(f_{x:\{1\}} \oplus g \oplus p\right), \tau\left(f_{x:\{0,1\}} \oplus g \oplus p\right), \tau(g \oplus p)\right) \\
& \geq M\left(\tau\left(f_{x:\{0\}} \oplus g \oplus p\right), \tau\left(f_{x:\{1\}} \oplus g \oplus p\right), \tau\left(f_{x:\{0,1\}} \oplus g\right)-1, \tau(g \oplus p)\right) \\
& \geq M\left(\tau\left(f_{x:\{0\}} \oplus g \oplus p\right), \tau\left(f_{x:\{1\}} \oplus g\right)-1, \tau\left(f_{x:\{0,1\}} \oplus g\right)-1, \tau(g \oplus p)\right)
\end{aligned}
$$

These values of $M$ can be checked during the computation of $T(f, g \oplus p)$, and $M \geq s+k-\tau(g \oplus p)$ implies the inequality (3). Thus the computation of $T(f, g \oplus p)$ can be broken off when $M \geq s+k-\tau(g \oplus p)$ holds. From the above discussion and Property 1 , we have a modified version of $T(f, g \oplus p)$ that has the breaking conditions. The procedure is shown as $T_{a}(f, g, p)$ in Fig. 3. Although $T_{a}$ has additional 
arguments to receive the values of $\tau\left(f_{x:\{1\}} \oplus g\right), \tau\left(f_{x:\{0,1\}} \oplus g\right)$, and $\tau(g \oplus p)$, the declaration of the arguments is omitted in Fig. 3 for simplicity of the description. A faster version of min-tau is obtained by replacing $T(f, g \oplus p)$ in min-tau with $T_{a}(f, g, p)$. The modified version is called min-tau $\beta 1$.

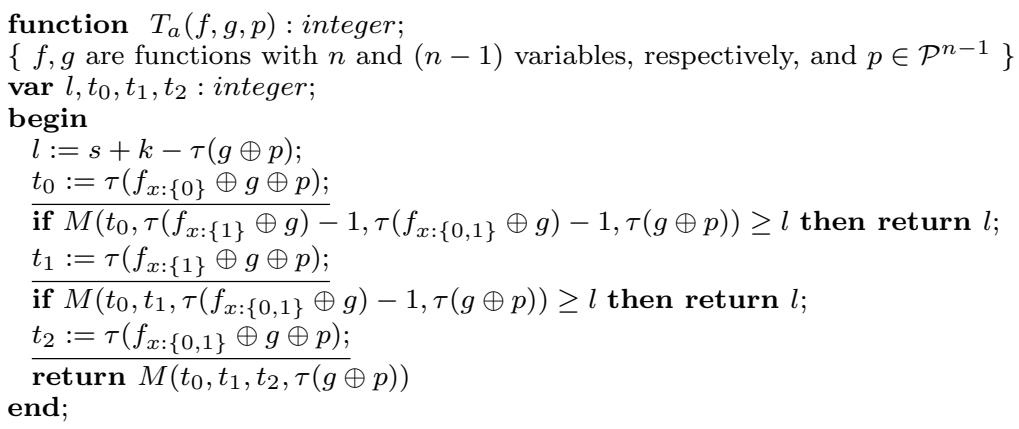

Fig. 3. $T_{a}: T(f, g \oplus p)$ with the breaking conditions

\section{2. $T(f, g \oplus p)$ Using Bounded Algorithm}

This section describes acceleration methods of $T_{a}$ by using an algorithm with upper bound specification, which has the optional argument $s_{u}$ to specify an upper bound of the return value of the algorithm (Fig. 4). The alternation is that " $s:=T(f, 0)$ " in Fig. 2, indicated by "*", is changed into an if form in Fig. 4. If the optional argument $s_{u}$ is specified, the value of $s_{u}$ will be the upper bound of min-tau $\left[s_{u}\right](f)$. Otherwise, $\min -\operatorname{tau}\left[s_{u}\right](f)$ will return the same value as min-tau $(f)$. From this property, min-tau $\left[s_{u}\right](f)$ in the case without specifying the optional argument $s_{u}$ is simply written as min-tau $(f)$ hereafter.

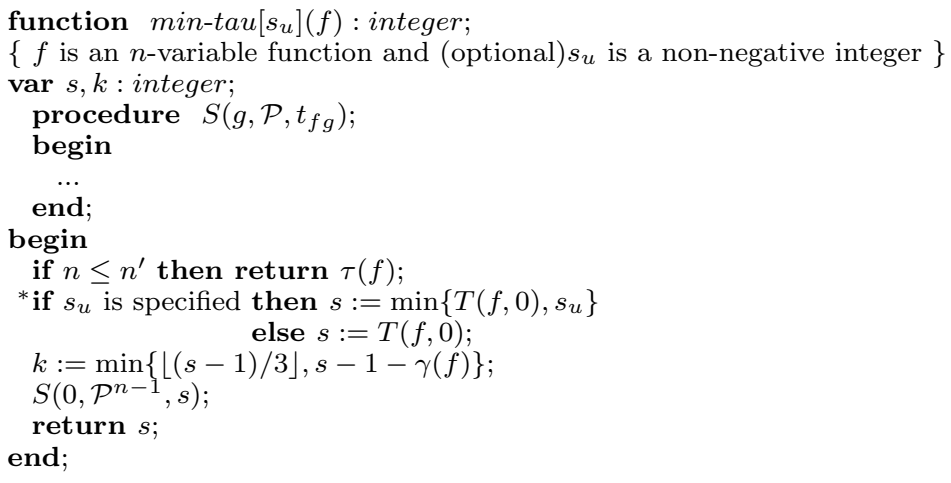

Fig. 4. min-tau with upper bound specification 
Function min-tau $\left[s_{u}\right](f)$ searches a smaller solution than $s_{u}$. If there exist such solutions, min-tau $\left[s_{u}\right](f)$ returns the minimum one among them. Otherwise, it returns $s_{u}$. This is summarized as the following property.

Property 2. Let $s_{u}$ be a non-negative integer.

$$
\text { min-tau }\left[s_{u}\right](f)=\left\{\begin{array}{r}
\tau(f)\left(\text { if } \tau(f)<s_{u}\right) \\
s_{u}\left(\text { if } \tau(f) \geq s_{u}\right)
\end{array}\right.
$$

Since the initial value of $s$ in $\min$-tau $\left[s_{u}\right](f)$ is $\min \left\{T(f, 0), s_{u}\right\}$, the search space of min-tau $\left[s_{u}\right](f)$ is not larger than that of min-tau $(f)$, that is, the computation time of min-tau $\left[s_{u}\right](f)$ is smaller or equal to that of min-tau $(f)$. Therefore, the computation time for $T(f, g \oplus p)$ is reduced if min-tau $\left[s_{u}\right]$ can be used instead of min-tau to obtain the size of subfunctions required in $T(f, g \oplus p)$. From this idea, $T_{a}$ in Fig. 3 is modified to $T_{b}$ in Fig. 5 , where the underlined steps in Fig. 3 are changed into if forms using min-tau $\left[s_{u}\right]$.

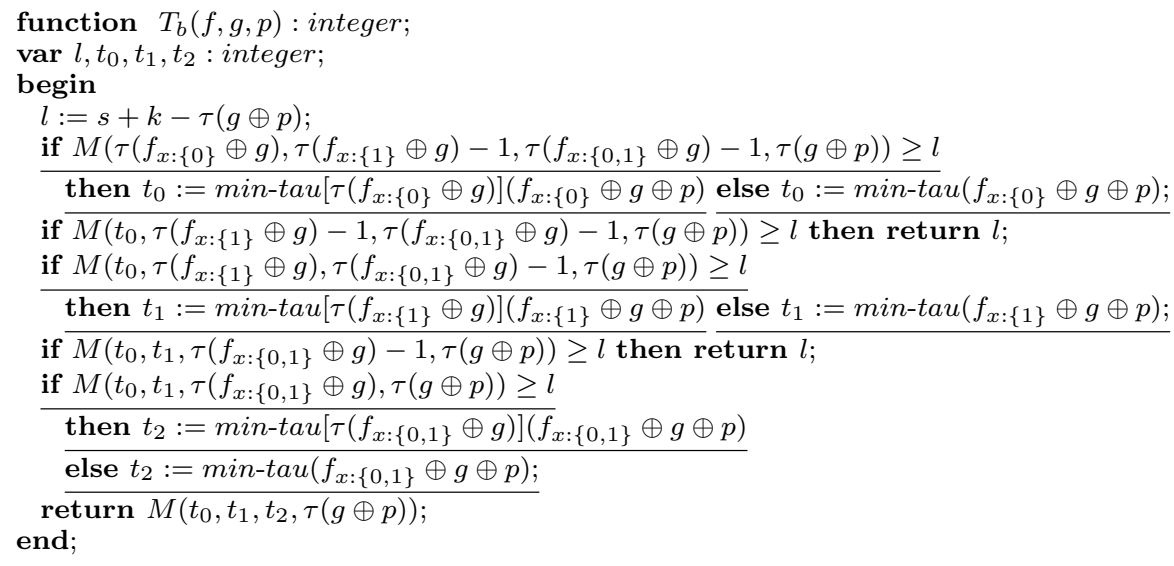

Fig. 5. $T_{b}: T(f, g \oplus p)$ using min-tau[ $\left[s_{u}\right]$

$T_{b}$ in Fig. 5 has three modified parts. Since these modifications are similar, we explain mainly the first underlined part. The step is to obtain $t_{0}$. There are three cases in the computation of the step as follows, where $l_{0}=M\left(\tau\left(f_{x:\{0\}} \oplus\right.\right.$ $\left.g), \tau\left(f_{x:\{1\}} \oplus g\right)-1, \tau\left(f_{x:\{0,1\}} \oplus g\right)-1, \tau(g \oplus p)\right)$.

Case 1 In the case of $l_{0}<l, t_{0}=\tau\left(f_{x:\{0\}} \oplus g \oplus p\right)$ is obtained at the else clause.

Case 2 In the case of $l_{0} \geq l$ and $\tau\left(f_{x:\{0\}} \oplus g \oplus p\right)<\tau\left(f_{x:\{0\}} \oplus g\right), t_{0}=\tau\left(f_{x:\{0\}} \oplus g \oplus p\right)$ is obtained from Property 2.

Case 3 In the case of $l_{0} \geq l$ and $\tau\left(f_{x:\{0\}} \oplus g \oplus p\right) \geq \tau\left(f_{x:\{0\}} \oplus g\right), t_{0}=\tau\left(f_{x:\{0\}} \oplus g\right)$ is obtained from Property 2.

While $t_{0}$ in Cases 1 and 2 results in $\tau\left(f_{x:\{0\}} \oplus g \oplus p\right)$ as well as $T_{a}, t_{0}$ in Case 3 results in $\tau\left(f_{x:\{0\}} \oplus g\right)$. In Case $3, M\left(\tau\left(f_{x:\{0\}} \oplus g \oplus p\right), \tau\left(f_{x:\{1\}} \oplus g\right)-1, \tau\left(f_{x:\{0,1\}} \oplus\right.\right.$ 
$g)-1, \tau(g \oplus p)) \geq M\left(t_{0}, \tau\left(f_{x:\{1\}} \oplus g\right)-1, \tau\left(f_{x:\{0,1\}} \oplus g\right)-1, \tau(g \oplus p)\right) \geq l$ holds from the assumption, and $T_{b}$ returns $l$ at the second if form. From the above inequality and the lower bound (7), the return value of $T_{b}$ satisfies Property 1 . The second and the third underlined parts, obtaining $t_{1}$ and $t_{2}$, can be discussed similarly. Thus $T_{b}$ can be used instead of $T_{a}$.

Among the three cases in the first modified part, the computation time is reduced particularly in Case 3 . The search space of min-tau $\left[\tau\left(f_{x:\{0\}} \oplus g\right)\right]\left(f_{x:\{0\}} \oplus g \oplus p\right)$ is smaller or equal to min-tau $\left(f_{x:\{0\}} \oplus g \oplus p\right)$ because $\tau\left(f_{x:\{0\}} \oplus g \oplus p\right) \geq \tau\left(f_{x:\{0\}} \oplus g\right)$ holds from the assumption of Case 3.

Although $T_{a}$ in Fig. 3 and $T_{b}$ in Fig. 5 compute the size of subfunctions in the order of $\tau\left(f_{x:\{0\}} \oplus g \oplus p\right), \tau\left(f_{x:\{1\}} \oplus g \oplus p\right)$, and $\tau\left(f_{x:\{0,1\}} \oplus g \oplus p\right)$, another order is also possible. One way for reducing the average computation time is to compute subfunctions in the increasing order of size. In general, minimization of a subfunction that has larger size tends to require a longer computation time. Minimization of such a subfunction will be omitted with a higher probability by leaving it in the later position. The problem is how to know the size of subfunctions before minimizing them. One method to predict the size of subfunctions is to check their lower bounds. In our real program, the computation order for $\tau\left(f_{x:\{0\}} \oplus g \oplus p\right)$, $\tau\left(f_{x:\{1\}} \oplus g \oplus p\right)$, and $\tau\left(f_{x:\{0,1\}} \oplus g \oplus p\right)$ is sorted according to their lower bounds (4)-(6).

The minimization algorithm using $T_{b}$ instead of $T$ in Fig. 4 is called min-tau $\beta 2$. One can obtain $\tau(f)$ by executing the algorithm without specifying the optional argument $s_{u}$.

In general, recursive calls consume memory as the call stack. The recursive calls of the procedure $S$ in our algorithms can be reduced by applying the so-called tail recursion optimization. The resulting code of min-tau $\beta 2$ is shown as min-tau 2 in Fig. 6, in which the recursive call $S\left(g, \mathcal{P}-\{p\}, t_{f g}\right)$ in the previous version has been rewritten in iteration, that is, the while statement. Although the call $S_{i t}\left(g \oplus p, \mathcal{P}, t_{f g p}\right)$ still remains recursive in the procedure, this modification has removed one of the most memory consuming problem in our algorithm. The maximum depth of the stack for $S\left(g, \mathcal{P}-\{p\}, t_{f g}\right)$ in the previous procedure depends on the cardinality of $\mathcal{P}\left(|\mathcal{P}| \leq\left|\mathcal{P}^{n-1}\right|=3^{n-1}\right)$, which is exponential with $n$. Meanwhile, that for $S_{i t}\left(g \oplus p, \mathcal{P}, t_{f g p}\right)$ depends on $k \approx \tau(f) / 3$, which is relatively small in the exact minimization.

\section{Multiple-Output Functions}

Practical circuits usually have multiple outputs. It is known that the minimization problem for multiple-output functions is equivalent to that for the special singleoutput functions called characteristic functions. ${ }^{18,29}$ By extending our algorithm to the characteristic functions, the exact minimum ESOPs of multiple-output functions can be obtained. Since the extension to the characteristic functions is a conventional technique, we omit the details. In order to make the paper self-contained, 


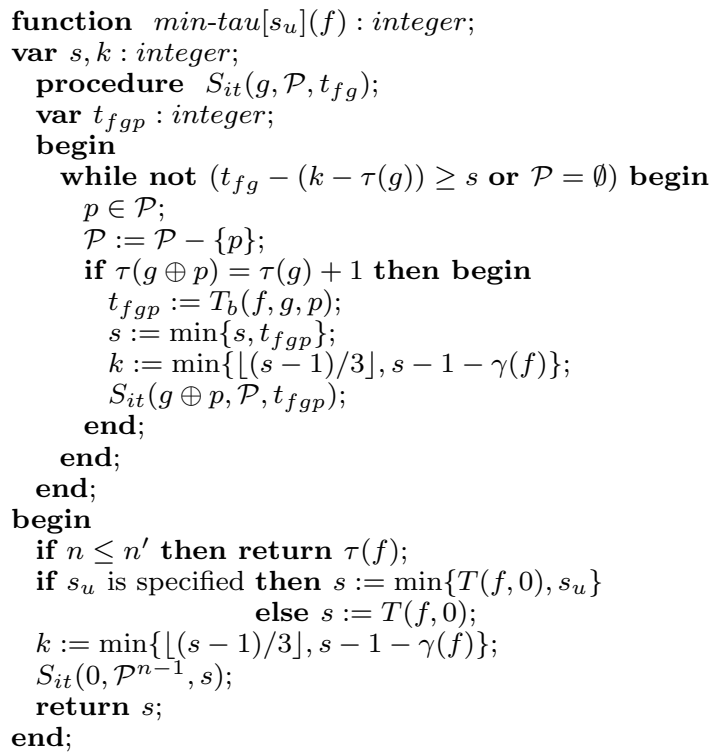

Fig. 6. min-tau2: a memory-efficient version of min-tau $\beta 2$

the outline is described in this section.

Let $M$ be a set $\{0,1, \cdots, m-1\}$ and $y$ be a variable of $M$. For a nonempty subset $S$ of $M, y^{S}$ is called a multiple-valued-input literal of $y$, which represents the following logic function with variable $y$.

$$
y^{S}=\left\{\begin{array}{l}
1 \text { (if } y \in S) \\
0(\text { if } y \notin S)
\end{array}\right.
$$

Let $M=\{0,1, \cdots, m-1\}$ and $B=\{0,1\}$. For a nonnegative integer $n$, a function $f: B^{n} \times M \rightarrow B$ is called an $(n, m)$-function. Let $\left(h_{0}, h_{1}, \cdots, h_{m-1}\right)$ be an $n$-input $m$-output function, where $h_{i}: B^{n} \rightarrow B$, i.e., $h_{i}$ is an ordinary $n$ variable logic function. The characteristic function $f$ of $\left(h_{0}, h_{1}, \cdots, h_{m-1}\right)$ is an $(n, m)$-function defined as follows.

$$
f\left(x_{n}, x_{n-1}, \cdots, x_{1}, y\right)=\bigoplus_{a \in M} h_{i} y^{\{a\}}
$$

The characteristic function $f$ is a single-output representation of the multipleoutput function, in which the output functions $\left(h_{0}, h_{1}, \cdots, h_{m-1}\right)$ are identified by $y$.

Example 3. Let $\left(h_{0}, h_{1}, h_{2}\right)$ be a 2-input 3-output function such that $h_{0}, h_{1}$, and $h_{2}$ are represented by $x_{2} x_{1}, x_{2} x_{1} \oplus \bar{x}_{2} \bar{x}_{1}$, and $\bar{x}_{2} x_{1} \oplus x_{2} \bar{x}_{1}$, respectively. The straightforward realization requires four product terms, in which $x_{2} x_{1}$ is shared between $h_{0}$ and $h_{1}$. These are minimum ESOPs for separate output functions. However, a collection of minimum ESOPs for separate outputs is not always a 
minimum ESOP for a multiple-output function. In fact, the characteristic function $f=h_{0} y^{\{0\}} \oplus h_{1} y^{\{1\}} \oplus h_{2} y^{\{2\}}$ can be represented by the following ESOP with three product terms.

$$
f=x_{2} x_{1} y^{\{0,1,2\}} \oplus \bar{x}_{2} \bar{x}_{1} y^{\{1,2\}} \oplus y^{\{2\}}
$$

A term $p \cdot y^{S}$ means that $p$ is the product term shared among the output functions $h_{i}$ such that $i \in S$. Hence, the 3 -output function $\left(h_{0}, h_{1}, h_{2}\right)$ is realized with three product terms as follows.

$$
\begin{aligned}
& h_{0}=x_{2} x_{1} \\
& h_{1}=x_{2} x_{1} \oplus \bar{x}_{2} \bar{x}_{1} \\
& h_{2}=x_{2} x_{1} \oplus \bar{x}_{2} \bar{x}_{1} \oplus 1
\end{aligned}
$$

In this way, the minimization problem for multiple-output functions can be reduced to that for $(n, m)$-functions.

The subfunctions of an $(n, m)$-function are defined in the same way as Definition 2. For an $(n, m)$-function $f$ and a variable $x$ of $B$, the subfunctions of $f$ with $x=0$ and $x=1$ are denoted by $f_{x:\{0\}}$ and $f_{x:\{1\}}$, respectively. $f_{x:\{0,1\}}$ is defined as $f_{x:\{0\}} \oplus f_{x:\{1\}}$. Note that these subfunctions are $(n-1, m)$-functions. By using these subfunctions, Eqs. (1) and (2) also hold for $(n, m)$-functions. Then we have the minimization theorem for $(n, m)$-functions. ${ }^{30}$

Theorem 2. For an $(n, m)$-function $f$ and a variable $x$ of $B, \tau(f)=\min \{T(f, g) \mid$ $\left.g \in \mathcal{F}^{(n-1, m)}\right\}$ holds, where $\mathcal{F}^{(n-1, m)}$ is the set of all $(n-1, m)$-functions.

Theorem 2 is the same as Theorem 1 in essence. The only difference is $\mathcal{F}^{n-1}$ in Theorem 1 and $\mathcal{F}^{(n-1, m)}$ in Theorem 2. Consequently, the minimization algorithms proposed in this paper are also applicable to $(n, m)$-functions by replacing $\mathcal{P}^{n-1}$ with $\mathcal{P}^{(n-1, m)}$, where $\mathcal{P}^{(n-1, m)}$ is the set of $(n-1, m)$-functions that can be represented by exactly one product. The cardinality of $\mathcal{P}^{(n-1, m)}$ is $3^{n-1} \cdot\left(2^{m}-1\right)$.

\section{Experimental Results}

\subsection{Obtaining Minimum ESOPs for MCNC PLA Benchmarks}

We implemented the original minimization algorithm min-tau and the proposed algorithms min-tau $\beta 1$ (Fig. 3), min-tau $\beta 2$, and min-tau2 (Fig. 6) in the C language. These programs were executed on a computer with an AMD Athlon64 3700+ $2.2 \mathrm{GHz}$ CPU and 1GB memory, whose operating system was Free BSD 6.2. By using the technique of characteristic functions, we obtained the minimum ESOPs of some functions in the MCNC PLA benchmark set, ${ }^{31}$ which has been widely used for evaluating ESOP-simplification algorithms. The minimum ESOPs of the MCNC benchmark functions had been unknown except for our preliminary work. ${ }^{35}$ Algorithm min-tau2 obtained minimum ESOPs of five functions: con1, misex1, rd53, sqrt8, and t481. The experimental results are shown in Tables 3,4 , and 5. 
Table 3 shows the computation time of our programs. The cells marked by "** mean that the programs could not obtain the results by execution for four days and gave up. These results demonstrate the fastness of min-tau $\beta 2$. Algorithm min-tau 2 is a stack-efficient version of min-tau $\beta 2$. Though the pattern of search, i.e., the order of choosing a product $p$ and testing the subfunctions $f_{x: I} \oplus g \oplus p$, is the same in min-tau $\beta 2$ and min-tau 2 in our realization, min-tau 2 is slightly faster than min-tau $\beta 2$ in Table 3. The improvement of time is considered as an indirect effect of the reduction of memory consumption. Because of the memory efficiency, only min-tau 2 obtained the minimum ESOP of t481. The "-" cells mean that the programs except for min-tau 2 could not obtain the results due to memory exhaustion. The comparison of max stack depth between min-tau $\beta 2$ and min-tau2 is shown in Table 4. In min-tau2, the consumption of stack is reduced significantly.

Table 5 shows a comparison with the state-of-the-art heuristic simplifiers, EXORCISM- $4{ }^{20}$ and QuiXOR. ${ }^{38}$ The column " $\# L$ " represents the number of literals of the resulting ESOP. For non-exact simplification, the number of products is represented by $\tau(F)$ instead of $\tau(f)$. Although the paper ${ }^{38}$ of QuiXOR did not provide the simplification results for t481, the related paper ${ }^{39}$ has given the ESOP of t481 with 13 products. The results of con1, misex1, rd53, and sqrt8 of EXORCISM4 are extracted from the literature ${ }^{38}$ since those results reported there were newer and better than the original. ${ }^{20}$ Our results show that these ESOPs obtained by EXORCISM- $4^{20}$ and QuiXOR ${ }^{38}$ are minimum ones. Note that there may be ESOPs consisting of fewer literals than our results because our minimization algorithms do not guarantee the minimality of the number of literals. In fact, QuiXOR has obtained the ESOP of misex1 that has fewer literals than our result. This confirms the superiority of QuiXOR. In comparison of computation time, min-tau2 is far slower than heuristic simplifiers. While min-tau 2 is valuable in theoretical aspects, EXORCISM-4 and QuiXOR are efficient as simplifiers in practice.

Table 3. Computation time for the MCNC PLA benchmark functions (seconds)

\begin{tabular}{ccrrrrr}
\hline Circuit & (in,out) & $\tau(f)$ & min-tau & min-tau $\beta 1$ & min-tau $\beta 2$ & min-tau2 \\
\hline con1 & $(7,2)$ & 9 & $*$ & 116.3 & 4.1 & 2.9 \\
misex1 & $(8,7)$ & 12 & $*$ & $*$ & 19697.0 & 8373.1 \\
rd53 & $(5,3)$ & 14 & 611.6 & 109.2 & 16.3 & 12.8 \\
sqrt8 & $(8,4)$ & 17 & $*$ & $*$ & 7857.4 & 6235.9 \\
t481 & $(16,1)$ & 13 & - & - & - & 1913.6 \\
\hline
\end{tabular}

\subsection{Minimization Results for Other Benchmarks}

In addition to the MCNC PLA benchmarks, min-tau 2 obtained the minimum ESOPs of some of other benchmarks. The brief results for them are given in this section. 
Table 4. Max stack depth for the MCNC PLA benchmark functions

\begin{tabular}{crr}
\hline Circuit & min-tauß32 & min-tau2 \\
\hline con1 & 2917 & 6 \\
misex1 & 277750 & 7 \\
rd53 & 757 & 8 \\
sqrt8 & 5364 & 9 \\
t481 & - & 4 \\
\hline
\end{tabular}

Table 5. Comparison with EXORCISM-4 and QuiXOR

\begin{tabular}{|c|c|c|c|c|c|c|c|c|c|}
\hline \multirow[b]{2}{*}{ Circuit } & \multicolumn{3}{|c|}{ EXORCISM- $4^{20}$} & \multicolumn{3}{|c|}{ QuiXOR $^{38}$} & \multicolumn{3}{|c|}{ min-tau 2} \\
\hline & $\tau(F)$ & $\# L$ & Time $[\mathrm{sec}]^{\dagger}$ & $\tau(F)$ & $\# L$ & Time $[\mathrm{sec}]^{\dagger}$ & $\tau(f)$ & $\# L$ & Time[sec] \\
\hline con1 & 9 & 28 & 0.33 & 9 & 28 & 7 & 9 & 28 & 2.9 \\
\hline misex1 & 12 & 48 & 1.39 & 12 & 46 & 77 & 12 & 48 & 8373.1 \\
\hline rd53 & 14 & 39 & 0.01 & 14 & 39 & 11 & 14 & 39 & 12.8 \\
\hline sqrt8 & 17 & 62 & 2.33 & 17 & 61 & 21 & 17 & 61 & 6235.9 \\
\hline $\mathrm{t} 481$ & 13 & 53 & $1^{\ddagger}$ & & & & 13 & 43 & 1913.6 \\
\hline
\end{tabular}

Table 6 shows the minimum results for the MCNC combinational benchmarks. $\mathrm{Cm} 42 \mathrm{a}$ required a longer computation time in spite of relatively smaller number of inputs. This is mainly because the benchmark has many outputs. The search space $\left|\mathcal{P}^{(n, m)}\right|$ increases exponentially with both $n$ and $m$.

Table 6. $\tau(f)$ for the MCNC combinational benchmark functions

\begin{tabular}{|c|c|c|c|c|c|c|c|}
\hline Circuit & (in,out) & $\tau(f)$ & Time [sec] & Circuit & (in,out) & $\tau(f)$ & Time [sec] \\
\hline $\mathrm{C} 17$ & $(5,2)$ & 6 & $<0.01$ & $\mathrm{~cm} 42 \mathrm{a}$ & $(4,10)$ & 11 & 475255.5 \\
\hline b1 & $(3,4)$ & 6 & $<0.01$ & $\mathrm{~cm} 82 \mathrm{a}$ & $(5,3)$ & 13 & 30.5 \\
\hline cm138a & $(6,8)$ & 9 & 1434.1 & majority & $(5,1)$ & 5 & $<0.01$ \\
\hline cm151a & $(12,2)$ & 9 & $<0.01$ & sqrt $8 \mathrm{ml}$ & $(8,4)$ & 8 & $<0.01$ \\
\hline cm152a & $(11,1)$ & 8 & $<0.01$ & $\mathrm{x} 2$ & $(10,7)$ & 15 & 4180.7 \\
\hline
\end{tabular}

Table 7 is the results for the arithmetic benchmarks, ${ }^{32}$ whose definitions are summarized in Table 8. For these arithmetic benchmarks, Koda-Sasao ${ }^{29}$ have given the size of ESOPs obtained by the heuristic simplification algorithm EXMIN2. ${ }^{18}$ Their results are cited in Table 7 as comparison; the computation time for those benchmarks was not presented in the paper. ${ }^{29}$ The minimum results for ADR2, MLP2, NRM2, and RDM4 have been known ${ }^{29}$ while those for larger benchmarks are presented in this paper. The most complex benchmark function that could be minimized by min-tau2 is NRM3, the size of which is 21 . Its very long computation time is due to the size. From Table 7, EXMIN2 produces minimum results for most benchmarks with $\tau(f) \leq 21$ except for NRM3, ROT4, ROT6, and SQR4. This confirms that EXMIN2 is an efficient simplifier in size of ESOPs. 
Table 7. $\tau(f)$ for the arithmetic benchmark functions and comparison with EXMIN2

\begin{tabular}{ccrrr}
\hline & & EXMIN2 & \multicolumn{2}{c}{ min-tau2 } \\
Circuit & $($ in,out $)$ & $\tau(F)$ & $\tau(f)$ & Time [sec] \\
\hline ADR2 & $(4,3)$ & 7 & 7 & $<0.01$ \\
ADR3 & $(6,4)$ & 15 & 15 & 27061.8 \\
INC4 & $(4,5)$ & 7 & 7 & $<0.01$ \\
INC5 & $(5,6)$ & & 9 & $<0.01$ \\
INC6 & $(6,7)$ & 11 & 11 & 0.20 \\
INC7 & $(7,8)$ & & 13 & 138.2 \\
INC8 & $(8,9)$ & & 15 & 73649.0 \\
LOG4 & $(4,4)$ & 10 & 10 & 0.24 \\
LOG5 & $(5,5)$ & & 16 & 281758.1 \\
MLP2 & $(4,4)$ & 5 & 5 & $<0.01$ \\
MLP3 & $(6,6)$ & 18 & 18 & 56258.3 \\
NRM2 & $(4,3)$ & 7 & 7 & $<0.01$ \\
NRM3 & $(6,4)$ & 26 & 21 & 1047388.4 \\
RDM4 & $(4,4)$ & 6 & 6 & $<0.01$ \\
RDM5 & $(5,5)$ & & 9 & $<0.01$ \\
RDM6 & $(6,6)$ & 15 & 15 & 107065.6 \\
ROT4 & $(4,3)$ & 8 & 7 & $<0.01$ \\
ROT5 & $(5,3)$ & & 11 & $<0.01$ \\
ROT6 & $(6,4)$ & 17 & 16 & 122.1 \\
SQR4 & $(4,8)$ & 15 & 11 & 583.3 \\
WGT4 & $(4,3)$ & 9 & 9 & $<0.01$ \\
WGT5 & $(5,3)$ & & 14 & 12.8 \\
\hline & & & &
\end{tabular}

Table 8. Arithmetic benchmark functions ${ }^{32}$

\begin{tabular}{lcl}
\hline Name & (in,out) & Function $\left(X, Y \in B^{n}\right)$ \\
\hline ADR $n$ & $(2 n, n+1)$ & $X+Y$ \\
INC $n$ & $(n, n+1)$ & $X+1$ \\
LOG $n$ & $(n, n)$ & $\left\lfloor\frac{2^{n}-1}{n} \times \log _{2}(X+1)\right\rfloor$ \\
MLP $n$ & $(2 n, 2 n)$ & $X \times Y$ \\
NRM $n$ & $(2 n, n+1)$ & $\left\lfloor\sqrt{X^{2}+Y^{2}}+0.5\right\rfloor$ \\
RDM $n$ & $(n, n)$ & $(5 X+1) \bmod 2^{n}$ \\
ROT $n$ & $(n,\lceil n / 2\rceil)$ & $\lfloor\sqrt{X}+0.5\rfloor$ \\
SQR $n$ & $(n, 2 n)$ & $X^{2}$ \\
WGT $n$ & $\left(n,\left\lceil\log _{2} n\right\rceil+1\right)$ & $\sum_{1 \leq i \leq n} x_{i}, X=\left(x_{1}, x_{2}, \ldots, x_{n}\right)$ \\
\hline
\end{tabular}

We obtained minimum ESOPs of all 7-variable symmetric functions, which are shown in Table 9 in Appendix. The notation $S_{n}^{A}$ represents the $n$-variable symmetric function that outputs 1 if and only if the arithmetic sum of the input values, i.e. $\sum_{1 \leq i \leq n} x_{i}$, is in $A$. In Table 9 , the representative functions of symmetric-Lequivalence $\operatorname{classes}^{28}$ are presented and the other functions are omitted because $\tau\left(f^{\prime}\right)=\tau(f)$ holds if $f^{\prime}$ is symmetric-L-equivalent to $f$. The notion of L-equivalence was originally introduced by Bioul-Davio-Deschamps ${ }^{36}$. The computation time depends on the size of functions; it took within 1 minute for functions with $\tau(f) \leq 15$, 


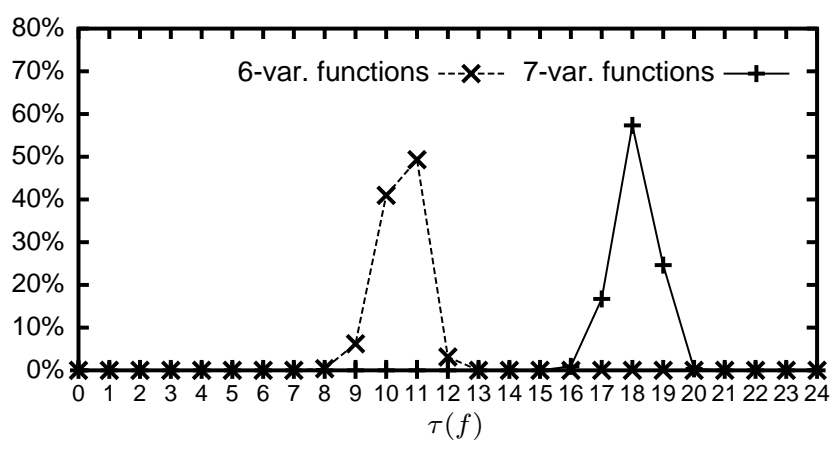

Fig. 7. Distributions of 6-variable functions (as dotted line) and 7-variable functions (as solid line)

within 1 hour for ones with $\tau(f) \leq 17$, within 2 weeks for ones with $\tau(f) \leq 21$, and within 4 weeks for ones with $\tau(f) \leq 24$. In 7 -variable symmetric functions, the function $S_{7}^{\{0,2,3,5,6\}}$ and its symmetric-L-equivalent functions $S_{7}^{\{1,2,4,5,7\}}$ and $S_{7}^{\{0,1,3,4,6,7\}}$ have the largest size of $\tau(f)=24$. The results are summarized as $\tau_{\max }\left(\mathcal{S}^{7}\right)=24$, where $\tau_{\max }\left(\mathcal{S}^{7}\right)$ is defined as $\max \left\{\tau(f) \mid f \in \mathcal{S}^{7}\right\}$ and $\mathcal{S}^{7}$ is the set of all 7 -variable symmetric functions. For $\tau_{\max }\left(\mathcal{S}^{7}\right)$, the lower bound ${ }^{25} 21 \leq \tau_{\max }\left(\mathcal{S}^{7}\right)$ and the upper bound ${ }^{9} \tau_{\max }\left(\mathcal{S}^{7}\right) \leq 27$ were given previously. These bounds are improved to $\tau_{\max }\left(\mathcal{S}^{7}\right)=24$ by our results. Similarly, the minimum ESOPs of Table 9 improve the constant factor of the upper and lower bounds ${ }^{9,25,33}$ on the size of symmetric functions. The size $\tau_{\max }\left(\mathcal{S}^{7}\right)=24$ is much smaller than the corresponding maximum size of AND-OR expressions for $\mathcal{S}^{7}$, which is 64 products for the parity function. We have also experimented on randomly-generated single-output functions and obtained the distributions of the size of 6- and 7-variable functions (Fig. 7). In the graph, the dotted line is for 6 -variable functions and the solid line is for 7 -variable functions. Most of randomly-generated functions with 7 variables are of $\tau(f)=17$ to 19 while symmetric functions contain many large-sized functions such that $\tau(f)=20$ or more. Symmetric functions tend to be more complex than randomly-generated functions. This suggests that symmetric functions can be used as complex single-output benchmarks in the minimization of AND-EXOR expressions.

\section{Conclusion}

We presented some acceleration methods for the exact minimization algorithm of ESOPs. They have reduced the computation in the time-consuming procedure in the algorithm by using the breaking conditions and the upper bound specification. With these methods, a new algorithm min-tau 2 was constructed. As the experimental results, min-tau 2 minimized many practical benchmark functions in the MCNC benchmarks, the arithmetic benchmarks, and 7-variable symmetric functions. The experiments have confirmed that min-tau 2 can minimize functions whose size is up 
to 10 in a practical computation time. For functions with a size of 11 or more, the computation time increases sharply with the size, and the extent of applicability is limited up to $(6,6)$-functions or 7 -variable single-output functions in practice. However, it is not a theoretical limitation. By taking a very long computation time, min-tau 2 obtained the exact minimum ESOPs of the 16-variable function t481 and complex symmetric functions with a size of 24 . These exact minimum results are valuable as the basic data for researches related to the EXOR-based logic synthesis since ESOPs are the most fundamental model of EXOR-based logic expressions.

\section{Appendix A. Minimum ESOPs of 7-variable symmetric functions}

Table 9: Minimum ESOPs of 7-variable symmetric functions

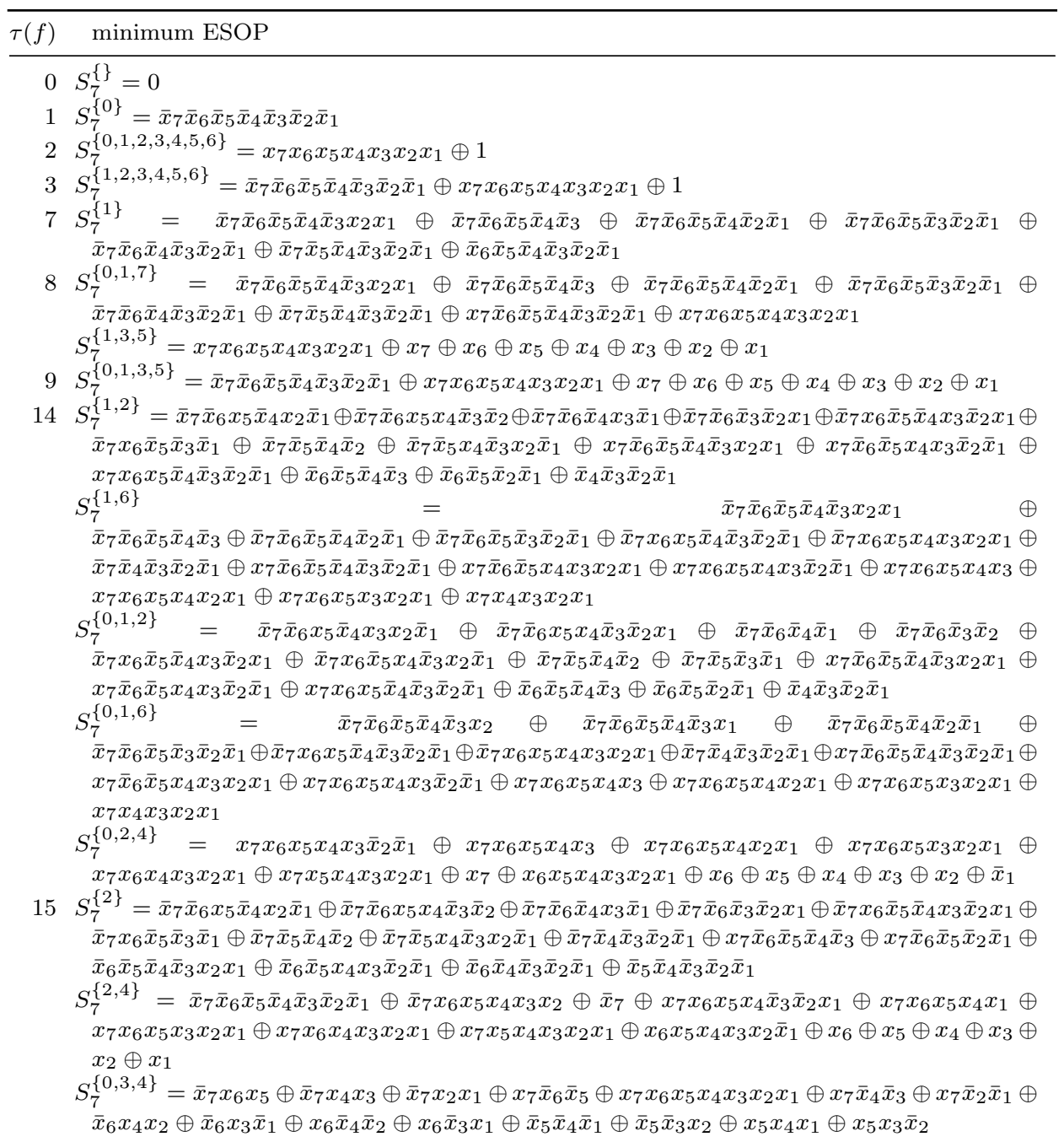


Table 9: Minimum ESOPs of 7-variable symmetric functions (continued)

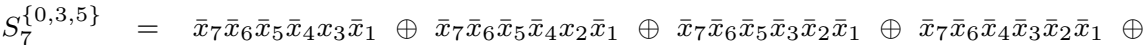
$\bar{x}_{7} \bar{x}_{5} \bar{x}_{4} \bar{x}_{3} \bar{x}_{2} \bar{x}_{1} \oplus x_{7} \bar{x}_{6} \bar{x}_{5} \bar{x}_{4} \bar{x}_{3} \bar{x}_{2} \oplus x_{7} x_{6} x_{5} x_{4} x_{3} x_{2} x_{1} \oplus x_{7} \oplus \bar{x}_{6} \bar{x}_{5} \bar{x}_{4} \bar{x}_{3} \bar{x}_{2} x_{1} \oplus \bar{x}_{6} \oplus x_{5} \oplus$ $x_{4} \oplus x_{3} \oplus x_{2} \oplus \bar{x}_{1}$

$S_{7}^{\{0,5,6\}}=\bar{x}_{7} \bar{x}_{6} \bar{x}_{5} \bar{x}_{4} \bar{x}_{3} \bar{x}_{2} \bar{x}_{1} \oplus \bar{x}_{7} \bar{x}_{6} \bar{x}_{5} x_{4} x_{3} x_{2} x_{1} \oplus \bar{x}_{7} x_{6} x_{5} \bar{x}_{4} \bar{x}_{3} x_{2} x_{1} \oplus \bar{x}_{7} x_{6} x_{5} x_{4} x_{3} \bar{x}_{2} \bar{x}_{1} \oplus$ $x_{7} \bar{x}_{6} x_{5} x_{4} \bar{x}_{3} x_{2} \bar{x}_{1} \oplus x_{7} \bar{x}_{6} x_{5} x_{3} x_{1} \oplus x_{7} x_{6} \bar{x}_{5} \bar{x}_{4} x_{3} x_{2} \oplus x_{7} x_{6} \bar{x}_{5} x_{4} \bar{x}_{2} x_{1} \oplus x_{7} x_{6} x_{4} \bar{x}_{3} x_{1} \oplus$ $x_{7} x_{6} x_{3} x_{2} \bar{x}_{1} \oplus x_{7} x_{5} \bar{x}_{4} x_{3} \bar{x}_{2} x_{1} \oplus x_{7} x_{5} x_{4} x_{2} \oplus x_{6} x_{5} x_{4} x_{3} \oplus x_{6} x_{5} x_{2} x_{1} \oplus x_{4} x_{3} x_{2} x_{1}$

$S_{7}^{\{0,1,3,5,6\}}=\bar{x}_{7} \bar{x}_{6} \bar{x}_{5} \bar{x}_{4} \bar{x}_{3} \bar{x}_{2} \bar{x}_{1} \oplus \bar{x}_{7} x_{6} x_{5} x_{4} x_{3} x_{2} \oplus \bar{x}_{7} \oplus x_{7} x_{6} x_{5} x_{4} x_{3} x_{1} \oplus x_{7} x_{6} x_{5} x_{4} x_{2} x_{1} \oplus$ $x_{7} x_{6} x_{5} x_{3} x_{2} x_{1} \oplus x_{7} x_{6} x_{4} x_{3} x_{2} x_{1} \oplus x_{7} x_{5} x_{4} x_{3} x_{2} x_{1} \oplus x_{6} x_{5} x_{4} x_{3} x_{2} \bar{x}_{1} \oplus x_{6} \oplus x_{5} \oplus x_{4} \oplus x_{3} \oplus$ $x_{2} \oplus \bar{x}_{1}$

$16 S_{7}^{\{0,5\}}=\bar{x}_{7} \bar{x}_{6} \bar{x}_{5} \bar{x}_{4} \bar{x}_{3} \bar{x}_{2} \bar{x}_{1} \oplus \bar{x}_{7} x_{6} x_{5} \bar{x}_{4} \bar{x}_{3} x_{2} x_{1} \oplus \bar{x}_{7} x_{6} x_{5} x_{4} x_{3} \bar{x}_{2} \bar{x}_{1} \oplus \bar{x}_{7} x_{6} x_{4} x_{3} x_{2} x_{1} \oplus$ $\bar{x}_{7} x_{5} x_{4} x_{3} x_{2} x_{1} \oplus x_{7} \bar{x}_{6} \bar{x}_{5} x_{4} x_{3} x_{2} x_{1} \oplus x_{7} \bar{x}_{6} x_{5} \bar{x}_{4} x_{3} \bar{x}_{2} x_{1} \oplus x_{7} \bar{x}_{6} x_{5} x_{4} \bar{x}_{3} x_{2} \bar{x}_{1} \oplus x_{7} x_{6} \bar{x}_{5} x_{4} x_{1} \oplus$ $x_{7} x_{6} \bar{x}_{5} x_{3} x_{2} \oplus x_{7} x_{6} \bar{x}_{4} x_{3} x_{2} \bar{x}_{1} \oplus x_{7} x_{6} x_{4} \bar{x}_{3} \bar{x}_{2} x_{1} \oplus x_{7} x_{5} x_{4} x_{2} \oplus x_{7} x_{5} x_{3} x_{1} \oplus x_{6} x_{5} x_{4} x_{3} \oplus$ $x_{6} x_{5} x_{2} x_{1}$

$S_{7}^{\{3,4\}}=\bar{x}_{7} \bar{x}_{6} \bar{x}_{5} \bar{x}_{4} \bar{x}_{3} \bar{x}_{2} \bar{x}_{1} \oplus \bar{x}_{7} \bar{x}_{6} \bar{x}_{5} \oplus \bar{x}_{7} \bar{x}_{4} \bar{x}_{3} \oplus \bar{x}_{7} \bar{x}_{2} \bar{x}_{1} \oplus x_{7} x_{6} x_{5} x_{4} x_{3} x_{2} x_{1} \oplus x_{7} x_{6} x_{5} \oplus$ $x_{7} x_{4} x_{3} \oplus x_{7} x_{2} x_{1} \oplus \bar{x}_{6} x_{4} x_{2} \oplus \bar{x}_{6} x_{3} x_{1} \oplus x_{6} \bar{x}_{4} \bar{x}_{2} \oplus x_{6} \bar{x}_{3} \bar{x}_{1} \oplus \bar{x}_{5} x_{4} x_{1} \oplus \bar{x}_{5} x_{3} x_{2} \oplus x_{5} \bar{x}_{4} \bar{x}_{1} \oplus$ $x_{5} \bar{x}_{3} \bar{x}_{2}$

$S_{7}^{\{0,2,7\}}=\bar{x}_{7} \bar{x}_{6} x_{5} \bar{x}_{4} x_{3} \bar{x}_{1} \quad \oplus \quad \bar{x}_{7} \bar{x}_{6} x_{5} \bar{x}_{3} \bar{x}_{2} x_{1} \quad \oplus$ $\bar{x}_{7} \bar{x}_{6} \bar{x}_{4} x_{2} \bar{x}_{1} \oplus \bar{x}_{7} \bar{x}_{6} x_{4} \bar{x}_{3} \bar{x}_{2} \oplus \bar{x}_{7} x_{6} \bar{x}_{5} \bar{x}_{4} x_{3} \bar{x}_{2} x_{1} \oplus \bar{x}_{7} x_{6} \bar{x}_{5} \bar{x}_{3} \bar{x}_{1} \oplus \bar{x}_{7} x_{6} x_{5} \bar{x}_{4} \bar{x}_{3} \bar{x}_{2} \bar{x}_{1} \oplus$ $\bar{x}_{7} \bar{x}_{5} \bar{x}_{4} \bar{x}_{2} \oplus \bar{x}_{7} \bar{x}_{5} x_{4} \bar{x}_{3} x_{2} \bar{x}_{1} \oplus x_{7} \bar{x}_{6} \bar{x}_{5} \bar{x}_{4} \bar{x}_{3} x_{2} x_{1} \oplus x_{7} \bar{x}_{6} \bar{x}_{5} x_{4} x_{3} \bar{x}_{2} \bar{x}_{1} \oplus x_{7} \bar{x}_{6} \bar{x}_{4} \bar{x}_{3} \bar{x}_{2} \bar{x}_{1} \oplus$ $x_{7} x_{6} x_{5} x_{4} x_{3} x_{2} x_{1} \oplus x_{7} \bar{x}_{5} \bar{x}_{4} \bar{x}_{3} \bar{x}_{2} \bar{x}_{1} \oplus \bar{x}_{6} \bar{x}_{5} \bar{x}_{4} \bar{x}_{3} \oplus \bar{x}_{6} \bar{x}_{5} \bar{x}_{2} \bar{x}_{1}$

$S_{7}^{\{0,3,4,5,6\}}=\bar{x}_{7} \bar{x}_{6} x_{5} \bar{x}_{4} x_{2} \bar{x}_{1} \oplus \bar{x}_{7} \bar{x}_{6} x_{5} x_{4} \bar{x}_{3} \bar{x}_{2} \oplus \bar{x}_{7} \bar{x}_{6} \bar{x}_{4} x_{3} \bar{x}_{1} \oplus \bar{x}_{7} \bar{x}_{6} \bar{x}_{3} \bar{x}_{2} x_{1} \oplus$ $\bar{x}_{7} x_{6} \bar{x}_{5} \bar{x}_{4} x_{3} \bar{x}_{2} x_{1} \oplus \bar{x}_{7} x_{6} \bar{x}_{5} \bar{x}_{3} \bar{x}_{1} \oplus \bar{x}_{7} \bar{x}_{5} \bar{x}_{4} \bar{x}_{2} \oplus \bar{x}_{7} \bar{x}_{5} x_{4} \bar{x}_{3} x_{2} \bar{x}_{1} \oplus x_{7} \bar{x}_{6} \bar{x}_{5} \bar{x}_{4} \bar{x}_{3} x_{2} x_{1} \oplus$ $x_{7} \bar{x}_{6} \bar{x}_{5} x_{4} x_{3} \bar{x}_{2} \bar{x}_{1} \oplus x_{7} x_{6} x_{5} \bar{x}_{4} \bar{x}_{3} \bar{x}_{2} \bar{x}_{1} \oplus x_{7} x_{6} x_{5} x_{4} x_{3} x_{2} x_{1} \oplus \bar{x}_{6} \bar{x}_{5} \bar{x}_{4} \bar{x}_{3} \oplus \bar{x}_{6} \bar{x}_{5} \bar{x}_{2} \bar{x}_{1} \oplus$ $\bar{x}_{4} \bar{x}_{3} \bar{x}_{2} \bar{x}_{1} \oplus 1$

$17 S_{7}^{\{0,2,3,6\}}=\bar{x}_{7} \bar{x}_{6} \bar{x}_{5} \bar{x}_{4} \bar{x}_{3} \bar{x}_{2} \bar{x}_{1} \oplus \bar{x}_{7} \bar{x}_{6} \bar{x}_{5} \oplus \bar{x}_{7} \bar{x}_{4} \bar{x}_{3} \oplus \bar{x}_{7} \bar{x}_{2} \bar{x}_{1} \oplus x_{7} x_{6} x_{5} x_{4} x_{3} x_{2} x_{1} \oplus x_{7} x_{6} x_{5} \oplus$ $x_{7} x_{4} x_{3} \oplus x_{7} x_{2} x_{1} \oplus x_{7} \oplus \bar{x}_{6} x_{4} x_{2} \oplus \bar{x}_{6} x_{3} \bar{x}_{1} \oplus x_{6} \bar{x}_{4} \bar{x}_{2} \oplus x_{6} \bar{x}_{3} x_{1} \oplus \bar{x}_{5} \bar{x}_{4} \bar{x}_{1} \oplus \bar{x}_{5} \bar{x}_{3} x_{2} \oplus$ $x_{5} x_{4} x_{1} \oplus x_{5} x_{3} \bar{x}_{2}$

$19 S_{7}^{\{3\}}=\bar{x}_{7} x_{6} \bar{x}_{5} \bar{x}_{2} \oplus \bar{x}_{7} x_{6} \bar{x}_{4} \bar{x}_{3} \oplus \bar{x}_{7} \bar{x}_{5} \bar{x}_{4} x_{3} x_{2} \bar{x}_{1} \oplus \bar{x}_{7} \bar{x}_{5} x_{4} x_{3} \bar{x}_{2} x_{1} \oplus \bar{x}_{7} x_{5} \bar{x}_{4} \bar{x}_{3} x_{2} x_{1} \oplus$ $\bar{x}_{7} x_{5} x_{4} \bar{x}_{3} \bar{x}_{2} \bar{x}_{1} \oplus x_{7} \bar{x}_{6} x_{5} x_{4} x_{3} x_{2} x_{1} \oplus x_{7} \bar{x}_{6} x_{5} x_{2} \oplus x_{7} \bar{x}_{6} x_{4} x_{3} \oplus x_{7} \bar{x}_{6} x_{1} \oplus x_{7} \bar{x}_{5} \bar{x}_{4} \bar{x}_{3} \bar{x}_{2} x_{1} \oplus$ $x_{7} \bar{x}_{5} x_{4} \bar{x}_{3} x_{2} \bar{x}_{1} \oplus x_{7} x_{5} \bar{x}_{4} x_{3} \bar{x}_{2} \bar{x}_{1} \oplus \bar{x}_{6} x_{5} x_{4} \oplus \bar{x}_{6} x_{5} x_{3} x_{1} \oplus \bar{x}_{6} x_{4} x_{2} x_{1} \oplus \bar{x}_{6} x_{3} x_{2} \oplus x_{6} \bar{x}_{5} \bar{x}_{3} \bar{x}_{1} \oplus$ $x_{6} \bar{x}_{4} \bar{x}_{2} \bar{x}_{1}$

$20 S_{7}^{\{0,3\}}=\bar{x}_{7} \bar{x}_{6} \bar{x}_{5} \bar{x}_{4} \bar{x}_{3} \bar{x}_{2} \bar{x}_{1} \oplus \bar{x}_{7} x_{6} \bar{x}_{5} \bar{x}_{2} \oplus \bar{x}_{7} x_{6} \bar{x}_{4} \bar{x}_{3} \oplus \bar{x}_{7} \bar{x}_{5} \bar{x}_{4} x_{3} x_{2} \bar{x}_{1} \oplus \bar{x}_{7} \bar{x}_{5} x_{4} x_{3} \bar{x}_{2} x_{1} \oplus$ $\bar{x}_{7} x_{5} \bar{x}_{4} \bar{x}_{3} x_{2} x_{1} \oplus \bar{x}_{7} x_{5} x_{4} \bar{x}_{3} \bar{x}_{2} \bar{x}_{1} \oplus x_{7} \bar{x}_{6} x_{5} x_{4} x_{3} x_{2} x_{1} \oplus x_{7} \bar{x}_{6} x_{5} x_{2} \oplus x_{7} \bar{x}_{6} x_{4} x_{3} \oplus x_{7} \bar{x}_{6} x_{1} \oplus$ $x_{7} \bar{x}_{5} \bar{x}_{4} \bar{x}_{3} \bar{x}_{2} x_{1} \oplus x_{7} \bar{x}_{5} x_{4} \bar{x}_{3} x_{2} \bar{x}_{1} \oplus x_{7} x_{5} \bar{x}_{4} x_{3} \bar{x}_{2} \bar{x}_{1} \oplus \bar{x}_{6} x_{5} x_{4} \oplus \bar{x}_{6} x_{5} x_{3} x_{1} \oplus \bar{x}_{6} x_{4} x_{2} x_{1} \oplus$ $\bar{x}_{6} x_{3} x_{2} \oplus x_{6} \bar{x}_{5} \bar{x}_{3} \bar{x}_{1} \oplus x_{6} \bar{x}_{4} \bar{x}_{2} \bar{x}_{1}$

$S_{7}^{\{1,3\}}=\bar{x}_{7} x_{6} x_{5} \bar{x}_{4} \bar{x}_{3} x_{2} x_{1} \oplus \bar{x}_{7} x_{6} x_{5} x_{4} x_{3} \bar{x}_{2} \bar{x}_{1} \oplus \bar{x}_{7} \bar{x}_{5} \bar{x}_{4} x_{3} x_{2} x_{1} \oplus \bar{x}_{7} \bar{x}_{5} \bar{x}_{3} x_{2} \bar{x}_{1} \oplus \bar{x}_{7} x_{5} \bar{x}_{4} \bar{x}_{3} \oplus$ $\bar{x}_{7} \bar{x}_{2} \bar{x}_{1} \oplus x_{7} \bar{x}_{6} x_{5} x_{4} x_{2} \oplus x_{7} \bar{x}_{6} x_{5} x_{3} x_{1} \oplus x_{7} \bar{x}_{6} x_{4} x_{1} \oplus x_{7} \bar{x}_{6} x_{3} x_{2} \oplus x_{7} x_{6} \bar{x}_{5} \bar{x}_{4} x_{3} x_{2} \bar{x}_{1} \oplus$ $x_{7} x_{6} \bar{x}_{5} x_{4} \bar{x}_{3} \bar{x}_{2} x_{1} \oplus x_{7} \bar{x}_{5} \bar{x}_{4} x_{1} \oplus x_{7} x_{5} \bar{x}_{4} \bar{x}_{3} \bar{x}_{2} \bar{x}_{1} \oplus \bar{x}_{6} x_{5} x_{4} x_{3} \oplus \bar{x}_{6} x_{5} x_{2} x_{1} \oplus \bar{x}_{6} x_{4} x_{3} x_{2} x_{1} \oplus$ $\bar{x}_{6} \oplus \bar{x}_{5} \bar{x}_{4} \oplus \bar{x}_{5} \bar{x}_{3} \bar{x}_{2}$

$S_{7}^{\{0,1,3\}}=\bar{x}_{7} x_{6} \bar{x}_{5} \bar{x}_{4} \bar{x}_{3} \bar{x}_{2} \bar{x}_{1} \oplus \bar{x}_{7} x_{6} x_{5} \bar{x}_{4} x_{3} \bar{x}_{2} x_{1} \oplus \bar{x}_{7} x_{6} x_{5} x_{4} \bar{x}_{3} x_{2} \bar{x}_{1} \oplus \bar{x}_{7} \bar{x}_{5} \bar{x}_{4} x_{3} x_{2} \bar{x}_{1} \oplus$ $\bar{x}_{7} \bar{x}_{5} x_{4} \bar{x}_{3} \bar{x}_{2} x_{1} \oplus \bar{x}_{7} \bar{x}_{4} \bar{x}_{2} \oplus \bar{x}_{7} \bar{x}_{3} \bar{x}_{1} \oplus x_{7} \bar{x}_{6} x_{5} x_{4} x_{1} \oplus x_{7} \bar{x}_{6} x_{5} x_{3} x_{2} \oplus x_{7} \bar{x}_{6} x_{4} x_{3} \oplus x_{7} \bar{x}_{6} x_{2} x_{1} \oplus$ $x_{7} x_{6} \bar{x}_{5} \bar{x}_{4} \bar{x}_{3} x_{2} x_{1} \oplus x_{7} x_{6} \bar{x}_{5} x_{4} x_{3} \bar{x}_{2} \bar{x}_{1} \oplus x_{7} x_{5} \bar{x}_{4} \bar{x}_{3} \bar{x}_{2} \bar{x}_{1} \oplus \bar{x}_{6} x_{5} x_{4} x_{2} \oplus \bar{x}_{6} x_{5} x_{3} x_{1} \oplus$ $\bar{x}_{6} x_{4} x_{3} x_{2} x_{1} \oplus \bar{x}_{6} \oplus \bar{x}_{5} \bar{x}_{4} \bar{x}_{3} \oplus \bar{x}_{5} \bar{x}_{2} \bar{x}_{1}$

$S_{7}^{\{0,1,5\}}=\bar{x}_{7} \bar{x}_{6} \bar{x}_{5} \bar{x}_{4} \oplus \bar{x}_{7} \bar{x}_{6} \bar{x}_{3} \bar{x}_{2} \oplus \bar{x}_{7} \bar{x}_{6} \oplus \bar{x}_{7} x_{6} \bar{x}_{1} \oplus \bar{x}_{7} \bar{x}_{5} \bar{x}_{3} \bar{x}_{1} \oplus \bar{x}_{7} x_{5} x_{2} \oplus \bar{x}_{7} \bar{x}_{4} \bar{x}_{2} \bar{x}_{1} \oplus$ $\bar{x}_{7} x_{4} x_{3} \oplus x_{7} \bar{x}_{6} \bar{x}_{5} \bar{x}_{4} \bar{x}_{3} \bar{x}_{2} \bar{x}_{1} \oplus x_{7} x_{6} x_{5} x_{4} \oplus x_{7} x_{6} x_{3} x_{2} \oplus x_{7} x_{5} x_{3} x_{1} \oplus x_{7} x_{4} x_{2} x_{1} \oplus$ $\bar{x}_{6} \bar{x}_{5} x_{4} \bar{x}_{3} x_{2} x_{1} \oplus \bar{x}_{6} x_{5} \bar{x}_{4} x_{3} \bar{x}_{2} x_{1} \oplus \bar{x}_{6} x_{5} x_{4} x_{3} x_{2} \bar{x}_{1} \oplus x_{6} \bar{x}_{5} \bar{x}_{4} x_{3} x_{2} \bar{x}_{1} \oplus x_{6} \bar{x}_{5} x_{4} x_{3} \bar{x}_{2} x_{1} \oplus$ $x_{6} x_{5} \bar{x}_{4} \bar{x}_{3} x_{2} x_{1} \oplus x_{6} x_{5} x_{4} \bar{x}_{3} \bar{x}_{2} \bar{x}_{1}$

$S_{7}^{\{0,3,7\}}=\bar{x}_{7} \bar{x}_{6} \bar{x}_{5} \bar{x}_{4} \bar{x}_{3} \bar{x}_{2} \bar{x}_{1} \oplus \bar{x}_{7} x_{6} \bar{x}_{5} \bar{x}_{2} \oplus \bar{x}_{7} x_{6} \bar{x}_{4} \bar{x}_{3} \oplus \bar{x}_{7} \bar{x}_{5} \bar{x}_{4} x_{3} x_{2} \bar{x}_{1} \oplus \bar{x}_{7} \bar{x}_{5} x_{4} x_{3} \bar{x}_{2} x_{1} \oplus$ $\bar{x}_{7} x_{5} \bar{x}_{4} \bar{x}_{3} x_{2} x_{1} \oplus \bar{x}_{7} x_{5} x_{4} \bar{x}_{3} \bar{x}_{2} \bar{x}_{1} \oplus x_{7} \bar{x}_{6} x_{5} x_{2} \oplus x_{7} \bar{x}_{6} x_{4} x_{3} \oplus x_{7} \bar{x}_{6} x_{1} \oplus x_{7} \bar{x}_{5} \bar{x}_{4} \bar{x}_{3} \bar{x}_{2} x_{1} \oplus$ $x_{7} \bar{x}_{5} x_{4} \bar{x}_{3} x_{2} \bar{x}_{1} \oplus x_{7} x_{5} \bar{x}_{4} x_{3} \bar{x}_{2} \bar{x}_{1} \oplus x_{7} x_{5} x_{4} x_{3} x_{2} x_{1} \oplus \bar{x}_{6} x_{5} x_{4} \oplus \bar{x}_{6} x_{5} x_{3} x_{1} \oplus \bar{x}_{6} x_{4} x_{2} x_{1} \oplus$ $\bar{x}_{6} x_{3} x_{2} \oplus x_{6} \bar{x}_{5} \bar{x}_{3} \bar{x}_{1} \oplus x_{6} \bar{x}_{4} \bar{x}_{2} \bar{x}_{1}$ 
Table 9: Minimum ESOPs of 7-variable symmetric functions (continued)

$S_{7}^{\{0,1,2,3,5\}}=\bar{x}_{7} \bar{x}_{6} \bar{x}_{5} \bar{x}_{4} x_{3} x_{2} x_{1} \oplus \bar{x}_{7} \bar{x}_{6} x_{5} x_{4} \bar{x}_{3} \bar{x}_{2} x_{1} \oplus \bar{x}_{7} x_{6} \bar{x}_{5} x_{4} \bar{x}_{3} x_{2} \bar{x}_{1} \oplus \bar{x}_{7} x_{6} x_{5} \bar{x}_{4} x_{3} \bar{x}_{2} \bar{x}_{1} \oplus$ $\bar{x}_{7} x_{6} x_{5} x_{4} x_{3} x_{2} x_{1} \oplus \bar{x}_{7} \oplus x_{7} \bar{x}_{6} \bar{x}_{5} \bar{x}_{4} \oplus x_{7} \bar{x}_{6} \bar{x}_{5} \bar{x}_{2} \bar{x}_{1} \oplus x_{7} \bar{x}_{6} \bar{x}_{4} \bar{x}_{3} \bar{x}_{1} \oplus x_{7} \bar{x}_{6} \bar{x}_{3} \bar{x}_{2} \oplus x_{7} \bar{x}_{5} \bar{x}_{4} \bar{x}_{3} \bar{x}_{2} \oplus$ $x_{7} \bar{x}_{5} \bar{x}_{3} \bar{x}_{1} \oplus x_{7} \bar{x}_{4} \bar{x}_{2} \bar{x}_{1} \oplus \bar{x}_{6} x_{5} x_{4} x_{3} x_{2} \bar{x}_{1} \oplus x_{6} \bar{x}_{5} x_{4} x_{3} \bar{x}_{2} x_{1} \oplus x_{6} x_{5} \bar{x}_{4} \bar{x}_{3} x_{2} x_{1} \oplus x_{6} x_{5} x_{3} \oplus$ $x_{6} x_{4} x_{2} \oplus x_{5} x_{4} x_{1} \oplus x_{3} x_{2} x_{1}$

$21 S_{7}^{\{0,2,6\}}=\bar{x}_{7} x_{6} x_{5} x_{3} \oplus \bar{x}_{7} x_{6} x_{4} x_{2} \oplus \bar{x}_{7} x_{6} \oplus \bar{x}_{7} \bar{x}_{5} \bar{x}_{4} \oplus \bar{x}_{7} \bar{x}_{5} \bar{x}_{2} \bar{x}_{1} \oplus \bar{x}_{7} \bar{x}_{4} \bar{x}_{3} \bar{x}_{1} \oplus$ $\bar{x}_{7} \bar{x}_{3} \bar{x}_{2} \oplus x_{7} \bar{x}_{6} \bar{x}_{5} \bar{x}_{4} \bar{x}_{3} \bar{x}_{2} \bar{x}_{1} \oplus x_{7} \bar{x}_{6} \bar{x}_{5} \bar{x}_{3} \oplus x_{7} \bar{x}_{6} \bar{x}_{4} \bar{x}_{2} \oplus x_{7} \bar{x}_{6} x_{1} \oplus x_{7} x_{5} x_{2} x_{1} \oplus x_{7} x_{4} x_{3} x_{1} \oplus$ $\bar{x}_{6} \bar{x}_{5} \bar{x}_{4} x_{3} x_{2} x_{1} \oplus \bar{x}_{6} \bar{x}_{5} x_{4} \bar{x}_{3} x_{2} \bar{x}_{1} \oplus \bar{x}_{6} x_{5} \bar{x}_{4} x_{3} \bar{x}_{2} \bar{x}_{1} \oplus \bar{x}_{6} x_{5} x_{4} \bar{x}_{3} \bar{x}_{2} x_{1} \oplus x_{6} \bar{x}_{5} x_{4} x_{3} \bar{x}_{2} x_{1} \oplus$ $x_{6} x_{5} \bar{x}_{4} \bar{x}_{3} x_{2} x_{1} \oplus x_{6} x_{5} x_{4} x_{3} x_{2} \bar{x}_{1} \oplus \bar{x}_{5} \bar{x}_{4} \bar{x}_{3} \bar{x}_{2} \bar{x}_{1}$

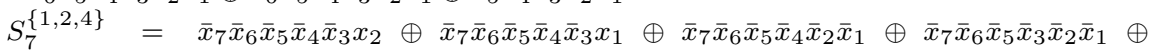
$\bar{x}_{7} \bar{x}_{6} \bar{x}_{4} \bar{x}_{3} \bar{x}_{2} \bar{x}_{1} \oplus \bar{x}_{7} \bar{x}_{5} \bar{x}_{4} \bar{x}_{3} \bar{x}_{2} \bar{x}_{1} \oplus x_{7} x_{6} x_{5} x_{4} x_{3} \bar{x}_{2} \bar{x}_{1} \oplus x_{7} x_{6} x_{5} x_{4} x_{3} \oplus x_{7} x_{6} x_{5} x_{4} x_{2} x_{1} \oplus$ $x_{7} x_{6} x_{5} x_{3} x_{2} x_{1} \oplus x_{7} x_{6} x_{4} x_{3} x_{2} x_{1} \oplus x_{7} x_{5} x_{4} x_{3} x_{2} x_{1} \oplus x_{7} \oplus \bar{x}_{6} \bar{x}_{5} \bar{x}_{4} \bar{x}_{3} \bar{x}_{2} \bar{x}_{1} \oplus \bar{x}_{6} \oplus$ $x_{6} x_{5} x_{4} x_{3} x_{2} x_{1} \oplus x_{5} \oplus x_{4} \oplus x_{3} \oplus x_{2} \oplus x_{1}$

$S_{7}^{\{1,2,5\}}=\bar{x}_{7} \bar{x}_{6} x_{5} x_{4} x_{3} x_{2} x_{1} \oplus \bar{x}_{7} x_{6} x_{5} x_{4} \bar{x}_{3} x_{2} \oplus \bar{x}_{7} x_{6} x_{5} x_{3} \bar{x}_{2} x_{1} \oplus \bar{x}_{7} \bar{x}_{4} \bar{x}_{3} \oplus \bar{x}_{7} x_{2} \bar{x}_{1} \oplus$ $x_{7} x_{6} x_{5} \bar{x}_{4} x_{3} x_{1} \oplus x_{7} x_{6} x_{5} x_{4} x_{2} \bar{x}_{1} \oplus x_{7} x_{6} x_{4} x_{3} x_{2} x_{1} \oplus x_{7} x_{6} \oplus x_{7} x_{5} \oplus x_{7} x_{4} x_{3} \oplus x_{7} \bar{x}_{2} x_{1} \oplus$ $\bar{x}_{6} \bar{x}_{5} \bar{x}_{3} \bar{x}_{2} \oplus \bar{x}_{6} x_{5} \bar{x}_{4} \bar{x}_{3} x_{2} \oplus \bar{x}_{6} x_{5} x_{3} \bar{x}_{2} \bar{x}_{1} \oplus \bar{x}_{6} x_{4} \bar{x}_{1} \oplus x_{6} \bar{x}_{5} x_{3} x_{2} \oplus x_{6} \bar{x}_{4} x_{1} \oplus \bar{x}_{5} \bar{x}_{4} \bar{x}_{2} \oplus \bar{x}_{5} \bar{x}_{3} \bar{x}_{1} \oplus$ $x_{5} \bar{x}_{4} x_{3} x_{2} \bar{x}_{1}$

$S_{7}^{\{1,2,6\}}=\bar{x}_{7} \bar{x}_{6} \bar{x}_{4} \bar{x}_{1} \oplus \bar{x}_{7} \bar{x}_{6} \bar{x}_{3} \bar{x}_{2} \oplus \bar{x}_{7} x_{6} \bar{x}_{5} \bar{x}_{4} x_{3} \bar{x}_{2} x_{1} \oplus \bar{x}_{7} x_{6} \bar{x}_{5} x_{4} \bar{x}_{3} x_{2} \bar{x}_{1} \oplus \bar{x}_{7} x_{6} \bar{x}_{4} \bar{x}_{3} \bar{x}_{2} \bar{x}_{1} \oplus$ $\bar{x}_{7} \bar{x}_{5} \bar{x}_{4} \bar{x}_{2} \oplus \bar{x}_{7} \bar{x}_{5} \bar{x}_{3} \bar{x}_{1} \oplus \bar{x}_{7} x_{5} x_{4} x_{3} x_{2} x_{1} \oplus x_{7} \bar{x}_{6} \bar{x}_{5} \bar{x}_{4} \bar{x}_{3} x_{2} x_{1} \oplus x_{7} \bar{x}_{6} \bar{x}_{5} x_{4} x_{3} \bar{x}_{2} \bar{x}_{1} \oplus$ $x_{7} x_{6} \bar{x}_{5} x_{4} x_{3} x_{2} x_{1} \oplus x_{7} x_{6} x_{5} x_{4} x_{1} \oplus x_{7} x_{6} x_{5} x_{3} x_{2} \oplus x_{7} \bar{x}_{5} \bar{x}_{4} \bar{x}_{3} \bar{x}_{2} \bar{x}_{1} \oplus x_{7} x_{5} \bar{x}_{4} x_{3} x_{2} \bar{x}_{1} \oplus$ $x_{7} x_{5} x_{4} \bar{x}_{3} \bar{x}_{2} x_{1} \oplus \bar{x}_{6} x_{5} x_{4} x_{2} \oplus \bar{x}_{6} x_{5} x_{3} x_{1} \oplus \bar{x}_{6} x_{5} \oplus \bar{x}_{6} \bar{x}_{4} \bar{x}_{3} \oplus \bar{x}_{6} \bar{x}_{2} \bar{x}_{1}$

$S_{7}^{\{0,1,2,6\}}=\bar{x}_{7} \bar{x}_{6} \bar{x}_{4} \bar{x}_{1} \oplus \bar{x}_{7} \bar{x}_{6} \bar{x}_{3} \bar{x}_{2} \oplus \bar{x}_{7} x_{6} \bar{x}_{5} \bar{x}_{4} \bar{x}_{2} \oplus \bar{x}_{7} x_{6} \bar{x}_{5} \bar{x}_{3} \bar{x}_{1} \oplus \bar{x}_{7} x_{6} x_{4} x_{3} x_{2} x_{1} \oplus$ $\bar{x}_{7} \bar{x}_{5} \bar{x}_{4} x_{3} \bar{x}_{2} x_{1} \oplus \bar{x}_{7} \bar{x}_{5} x_{4} \bar{x}_{3} x_{2} \bar{x}_{1} \oplus \bar{x}_{7} x_{5} \bar{x}_{4} \bar{x}_{3} \bar{x}_{2} \bar{x}_{1} \oplus x_{7} \bar{x}_{6} x_{5} \oplus x_{7} \bar{x}_{6} \bar{x}_{4} \bar{x}_{3} \oplus x_{7} \bar{x}_{6} \bar{x}_{2} \bar{x}_{1} \oplus$ $x_{7} x_{6} x_{5} \bar{x}_{4} x_{3} \bar{x}_{2} x_{1} \oplus x_{7} x_{6} x_{5} x_{4} \bar{x}_{3} x_{2} \bar{x}_{1} \oplus x_{7} x_{5} x_{4} x_{2} \oplus x_{7} x_{5} x_{3} x_{1} \oplus \bar{x}_{6} \bar{x}_{5} \bar{x}_{4} \bar{x}_{3} x_{2} x_{1} \oplus$ $\bar{x}_{6} \bar{x}_{5} x_{4} x_{3} \bar{x}_{2} \bar{x}_{1} \oplus \bar{x}_{6} x_{5} \bar{x}_{4} x_{3} x_{2} \bar{x}_{1} \oplus \bar{x}_{6} x_{5} x_{4} \bar{x}_{3} \bar{x}_{2} x_{1} \oplus x_{6} \bar{x}_{5} x_{4} x_{3} x_{2} x_{1} \oplus \bar{x}_{5} \bar{x}_{4} \bar{x}_{3} \bar{x}_{2} \bar{x}_{1}$

$S_{7}^{\{1,2,3,5\}}=\bar{x}_{7} \bar{x}_{6} \bar{x}_{5} \bar{x}_{4} \bar{x}_{3} \bar{x}_{2} \bar{x}_{1} \oplus \bar{x}_{7} \bar{x}_{6} \bar{x}_{5} \bar{x}_{4} x_{3} x_{2} x_{1} \oplus \bar{x}_{7} \bar{x}_{6} x_{5} x_{4} \bar{x}_{3} \bar{x}_{2} x_{1} \oplus \bar{x}_{7} x_{6} \bar{x}_{5} x_{4} \bar{x}_{3} x_{2} \bar{x}_{1} \oplus$ $\bar{x}_{7} x_{6} x_{5} \bar{x}_{4} x_{3} \bar{x}_{2} \bar{x}_{1} \oplus \bar{x}_{7} x_{6} x_{5} x_{4} x_{3} x_{2} x_{1} \oplus \bar{x}_{7} \oplus x_{7} \bar{x}_{6} \bar{x}_{5} \bar{x}_{4} \oplus x_{7} \bar{x}_{6} \bar{x}_{5} \bar{x}_{2} \bar{x}_{1} \oplus x_{7} \bar{x}_{6} \bar{x}_{4} \bar{x}_{3} \bar{x}_{1} \oplus$ $x_{7} \bar{x}_{6} \bar{x}_{3} \bar{x}_{2} \oplus x_{7} \bar{x}_{5} \bar{x}_{4} \bar{x}_{3} \bar{x}_{2} \oplus x_{7} \bar{x}_{5} \bar{x}_{3} \bar{x}_{1} \oplus x_{7} \bar{x}_{4} \bar{x}_{2} \bar{x}_{1} \oplus \bar{x}_{6} x_{5} x_{4} x_{3} x_{2} \bar{x}_{1} \oplus x_{6} \bar{x}_{5} x_{4} x_{3} \bar{x}_{2} x_{1} \oplus$ $x_{6} x_{5} \bar{x}_{4} \bar{x}_{3} x_{2} x_{1} \oplus x_{6} x_{5} x_{3} \oplus x_{6} x_{4} x_{2} \oplus x_{5} x_{4} x_{1} \oplus x_{3} x_{2} x_{1}$

$S_{7}^{\{0,1,2,3,6\}}=\bar{x}_{7} \bar{x}_{6} \bar{x}_{5} x_{4} \bar{x}_{3} x_{2} x_{1} \oplus \bar{x}_{7} \bar{x}_{6} x_{5} \bar{x}_{4} x_{3} \bar{x}_{2} x_{1} \oplus \bar{x}_{7} x_{6} \bar{x}_{5} \bar{x}_{4} x_{3} x_{2} \bar{x}_{1} \oplus \bar{x}_{7} x_{6} x_{5} x_{4} \bar{x}_{3} \bar{x}_{2} \bar{x}_{1} \oplus$ $\bar{x}_{7} x_{6} x_{5} x_{2} x_{1} \oplus \bar{x}_{7} x_{6} x_{4} x_{3} x_{1} \oplus \bar{x}_{7} x_{5} x_{4} x_{3} x_{2} \oplus x_{7} \bar{x}_{6} \bar{x}_{5} \bar{x}_{4} \bar{x}_{3} \bar{x}_{2} \bar{x}_{1} \oplus x_{7} \bar{x}_{6} \bar{x}_{5} \bar{x}_{4} \oplus x_{7} \bar{x}_{6} \bar{x}_{3} \bar{x}_{2} \oplus$ $x_{7} \bar{x}_{6} \bar{x}_{1} \oplus x_{7} \bar{x}_{5} \bar{x}_{3} \bar{x}_{1} \oplus x_{7} \bar{x}_{5} \bar{x}_{2} \oplus x_{7} \bar{x}_{4} \bar{x}_{3} \oplus x_{7} \bar{x}_{4} \bar{x}_{2} \bar{x}_{1} \oplus x_{6} x_{5} x_{4} x_{3} x_{2} x_{1} \oplus x_{6} x_{5} x_{4} \oplus x_{6} x_{3} x_{2} \oplus$ $x_{5} x_{3} x_{1} \oplus x_{4} x_{2} x_{1} \oplus 1$

$S_{7}^{\{0,1,2,4,7\}}=\bar{x}_{7} \bar{x}_{6} \bar{x}_{5} \bar{x}_{4} \bar{x}_{3} x_{2} \oplus \bar{x}_{7} \bar{x}_{6} \bar{x}_{5} \bar{x}_{4} \bar{x}_{3} x_{1} \oplus \bar{x}_{7} \bar{x}_{6} \bar{x}_{5} \bar{x}_{4} \bar{x}_{2} \bar{x}_{1} \oplus \bar{x}_{7} \bar{x}_{6} \bar{x}_{5} \bar{x}_{3} \bar{x}_{2} \bar{x}_{1} \oplus$ $\bar{x}_{7} x_{6} x_{5} \bar{x}_{4} \bar{x}_{3} \bar{x}_{2} \bar{x}_{1} \oplus \bar{x}_{7} x_{6} x_{5} x_{4} x_{3} x_{2} x_{1} \oplus \bar{x}_{7} \bar{x}_{4} \bar{x}_{3} \bar{x}_{2} \bar{x}_{1} \oplus x_{7} x_{6} x_{5} x_{4} x_{3} \bar{x}_{2} \bar{x}_{1} \oplus x_{7} x_{6} x_{5} x_{4} x_{3} \oplus$ $x_{7} x_{6} x_{5} x_{4} x_{2} x_{1} \oplus x_{7} x_{6} x_{5} x_{3} x_{2} x_{1} \oplus x_{7} x_{6} x_{4} x_{3} x_{2} x_{1} \oplus x_{7} x_{5} x_{4} x_{3} x_{2} x_{1} \oplus x_{7} \oplus \bar{x}_{6} \bar{x}_{5} \bar{x}_{4} \bar{x}_{3} \bar{x}_{2} \bar{x}_{1} \oplus$ $\bar{x}_{6} \oplus x_{5} \oplus x_{4} \oplus x_{3} \oplus x_{2} \oplus x_{1}$

$S_{7}^{\{0,1,3,4,7\}}=\bar{x}_{7} \bar{x}_{6} \bar{x}_{5} \bar{x}_{4} \bar{x}_{3} \bar{x}_{2} \oplus \bar{x}_{7} \bar{x}_{6} \bar{x}_{5} x_{4} \bar{x}_{3} x_{2} \bar{x}_{1} \oplus \bar{x}_{7} x_{6} x_{5} \bar{x}_{4} \bar{x}_{3} \bar{x}_{2} \bar{x}_{1} \oplus \bar{x}_{7} x_{6} \oplus \bar{x}_{7} x_{5} \oplus$ $\bar{x}_{7} \bar{x}_{4} x_{3} \oplus \bar{x}_{7} x_{4} \bar{x}_{3} \bar{x}_{2} \bar{x}_{1} \oplus x_{7} \bar{x}_{6} \bar{x}_{5} \bar{x}_{4} x_{3} \bar{x}_{2} \bar{x}_{1} \oplus x_{7} \bar{x}_{6} \bar{x}_{5} \bar{x}_{3} \bar{x}_{1} \oplus x_{7} x_{4} \bar{x}_{3} \oplus x_{7} x_{3} \bar{x}_{2} \bar{x}_{1} \oplus x_{7} x_{2} x_{1} \oplus$ $\bar{x}_{6} \bar{x}_{5} x_{3} x_{1} \oplus \bar{x}_{6} \bar{x}_{4} x_{1} \oplus \bar{x}_{6} x_{2} \oplus x_{6} \bar{x}_{5} \bar{x}_{4} x_{2} \bar{x}_{1} \oplus x_{6} x_{5} x_{4} \bar{x}_{1} \oplus x_{6} x_{5} \bar{x}_{3} \oplus \bar{x}_{5} \bar{x}_{4} \bar{x}_{2} x_{1} \oplus x_{5} x_{4} x_{2} \oplus$ $x_{3} x_{2} x_{1}$

$S_{7}^{\{0,1,4,5,6\}}=\bar{x}_{7} x_{6} \bar{x}_{5} \bar{x}_{4} \bar{x}_{1} \oplus \bar{x}_{7} x_{6} \bar{x}_{5} x_{4} \bar{x}_{2} x_{1} \oplus \bar{x}_{7} x_{6} x_{5} x_{4} \bar{x}_{3} x_{2} x_{1} \oplus \bar{x}_{7} x_{6} x_{5} \bar{x}_{3} x_{1} \oplus \bar{x}_{7} x_{6} \bar{x}_{3} \bar{x}_{2} \oplus$ $\bar{x}_{7} \bar{x}_{5} \bar{x}_{2} \oplus \bar{x}_{7} \bar{x}_{4} \bar{x}_{3} \oplus x_{7} \bar{x}_{6} x_{5} x_{2} \oplus x_{7} \bar{x}_{6} x_{4} x_{3} \oplus x_{7} \bar{x}_{6} x_{1} \oplus x_{7} x_{6} \bar{x}_{5} \bar{x}_{4} \bar{x}_{3} \bar{x}_{2} \oplus x_{7} x_{6} \bar{x}_{5} x_{4} \bar{x}_{3} x_{2} \bar{x}_{1} \oplus$ $x_{7} x_{6} x_{5} \bar{x}_{4} x_{3} \bar{x}_{2} \bar{x}_{1} \oplus x_{7} x_{5} x_{4} x_{3} x_{2} x_{1} \oplus \bar{x}_{6} x_{5} x_{4} \oplus \bar{x}_{6} x_{5} x_{3} x_{1} \oplus \bar{x}_{6} x_{4} x_{2} x_{1} \oplus \bar{x}_{6} x_{3} x_{2} \oplus \bar{x}_{5} \bar{x}_{3} \bar{x}_{1} \oplus$ $\bar{x}_{4} \bar{x}_{2} \bar{x}_{1} \oplus 1$

$S_{7}^{\{1,2,3,5,6\}}=\bar{x}_{7} \bar{x}_{6} \bar{x}_{5} \bar{x}_{3} \bar{x}_{2} \bar{x}_{1} \oplus \bar{x}_{7} \bar{x}_{6} x_{5} \bar{x}_{3} x_{2} x_{1} \oplus \bar{x}_{7} \bar{x}_{6} x_{4} \bar{x}_{2} \oplus \bar{x}_{7} x_{6} \bar{x}_{5} x_{3} \bar{x}_{2} x_{1} \oplus$ $\bar{x}_{7} x_{6} x_{5} x_{3} x_{2} \bar{x}_{1} \oplus \bar{x}_{7} \bar{x}_{5} x_{4} \bar{x}_{3} \oplus \bar{x}_{7} x_{4} \bar{x}_{1} \oplus x_{7} \bar{x}_{6} \bar{x}_{5} x_{3} x_{2} x_{1} \oplus x_{7} \bar{x}_{6} x_{5} x_{3} \bar{x}_{2} \bar{x}_{1} \oplus x_{7} x_{6} \bar{x}_{5} \bar{x}_{3} x_{2} \bar{x}_{1} \oplus$ $x_{7} x_{6} x_{5} x_{4} x_{3} x_{2} x_{1} \oplus x_{7} x_{6} x_{5} \bar{x}_{3} \bar{x}_{2} x_{1} \oplus x_{7} x_{6} \bar{x}_{4} x_{2} \oplus x_{7} x_{5} \bar{x}_{4} x_{3} \oplus \bar{x}_{6} \bar{x}_{5} x_{4} \oplus \bar{x}_{6} x_{4} \bar{x}_{3} \bar{x}_{1} \oplus$ $x_{6} \bar{x}_{4} x_{3} x_{1} \oplus \bar{x}_{5} x_{4} \bar{x}_{2} \bar{x}_{1} \oplus x_{5} \bar{x}_{4} x_{2} x_{1} \oplus x_{4} \bar{x}_{3} \bar{x}_{2} \oplus 1$ 
Table 9: Minimum ESOPs of 7-variable symmetric functions (continued)

$22 S_{7}^{\{1,4\}}=\bar{x}_{7} \bar{x}_{6} \bar{x}_{5} \bar{x}_{4} \bar{x}_{3} x_{2} x_{1} \oplus \bar{x}_{7} \bar{x}_{6} \bar{x}_{5} \bar{x}_{4} \bar{x}_{3} \oplus \bar{x}_{7} x_{6} x_{5} \bar{x}_{2} \bar{x}_{1} \oplus \bar{x}_{7} x_{6} x_{4} x_{2} \oplus \bar{x}_{7} x_{6} x_{3} x_{1} \oplus$ $\bar{x}_{7} x_{5} x_{4} x_{1} \oplus \bar{x}_{7} x_{5} x_{3} x_{2} \oplus \bar{x}_{7} x_{4} x_{3} \bar{x}_{2} \bar{x}_{1} \oplus x_{7} \bar{x}_{6} \bar{x}_{5} \oplus x_{7} \bar{x}_{6} \bar{x}_{4} \bar{x}_{2} x_{1} \oplus x_{7} \bar{x}_{6} \bar{x}_{3} x_{2} \bar{x}_{1} \oplus$ $x_{7} x_{6} x_{5} x_{4} x_{3} \bar{x}_{2} \bar{x}_{1} \oplus x_{7} \bar{x}_{5} \bar{x}_{4} x_{2} \bar{x}_{1} \oplus x_{7} \bar{x}_{5} \bar{x}_{3} \bar{x}_{2} x_{1} \oplus x_{7} \bar{x}_{4} \bar{x}_{3} \oplus \bar{x}_{6} \bar{x}_{5} x_{4} x_{3} x_{2} x_{1} \oplus \bar{x}_{6} x_{5} \bar{x}_{4} x_{3} \bar{x}_{1} \oplus$ $\bar{x}_{6} x_{5} x_{4} \bar{x}_{3} \bar{x}_{2} \oplus x_{6} \bar{x}_{5} \bar{x}_{4} x_{3} \bar{x}_{2} \oplus x_{6} \bar{x}_{5} x_{4} \bar{x}_{3} \bar{x}_{1} \oplus x_{6} x_{5} \bar{x}_{4} \bar{x}_{3} x_{2} x_{1} \oplus \bar{x}_{2} \bar{x}_{1}$

$S_{7}^{\{0,1,4\}}=\bar{x}_{7} \bar{x}_{6} \bar{x}_{5} \bar{x}_{4} \bar{x}_{3} x_{2} \oplus \bar{x}_{7} \bar{x}_{6} \bar{x}_{5} \bar{x}_{4} \bar{x}_{3} x_{1} \oplus \bar{x}_{7} x_{6} x_{5} \bar{x}_{2} \bar{x}_{1} \oplus \bar{x}_{7} x_{6} x_{4} x_{2} \oplus \bar{x}_{7} x_{6} x_{3} x_{1} \oplus$ $\bar{x}_{7} x_{5} x_{4} x_{1} \oplus \bar{x}_{7} x_{5} x_{3} x_{2} \oplus \bar{x}_{7} x_{4} x_{3} \bar{x}_{2} \bar{x}_{1} \oplus x_{7} \bar{x}_{6} \bar{x}_{5} \oplus x_{7} \bar{x}_{6} \bar{x}_{4} \bar{x}_{2} x_{1} \oplus x_{7} \bar{x}_{6} \bar{x}_{3} x_{2} \bar{x}_{1} \oplus$ $x_{7} x_{6} x_{5} x_{4} x_{3} \bar{x}_{2} \bar{x}_{1} \oplus x_{7} \bar{x}_{5} \bar{x}_{4} x_{2} \bar{x}_{1} \oplus x_{7} \bar{x}_{5} \bar{x}_{3} \bar{x}_{2} x_{1} \oplus x_{7} \bar{x}_{4} \bar{x}_{3} \oplus \bar{x}_{6} \bar{x}_{5} x_{4} x_{3} x_{2} x_{1} \oplus \bar{x}_{6} x_{5} \bar{x}_{4} x_{3} \bar{x}_{1} \oplus$ $\bar{x}_{6} x_{5} x_{4} \bar{x}_{3} \bar{x}_{2} \oplus x_{6} \bar{x}_{5} \bar{x}_{4} x_{3} \bar{x}_{2} \oplus x_{6} \bar{x}_{5} x_{4} \bar{x}_{3} \bar{x}_{1} \oplus x_{6} x_{5} \bar{x}_{4} \bar{x}_{3} x_{2} x_{1} \oplus \bar{x}_{2} \bar{x}_{1}$

$S_{7}^{\{1,3,4\}}=\bar{x}_{7} \bar{x}_{6} \bar{x}_{5} \bar{x}_{4} \bar{x}_{3} x_{2} \bar{x}_{1} \oplus \bar{x}_{7} \bar{x}_{6} \bar{x}_{5} x_{4} \bar{x}_{3} \bar{x}_{2} x_{1} \oplus \bar{x}_{7} x_{6} x_{5} \bar{x}_{4} \bar{x}_{3} \bar{x}_{2} \bar{x}_{1} \oplus \bar{x}_{7} x_{6} \oplus \bar{x}_{7} x_{5} \oplus$ $\bar{x}_{7} \bar{x}_{4} x_{3} \oplus \bar{x}_{7} x_{4} \bar{x}_{3} \bar{x}_{2} \bar{x}_{1} \oplus x_{7} \bar{x}_{6} \bar{x}_{5} \bar{x}_{4} x_{3} \bar{x}_{2} \bar{x}_{1} \oplus x_{7} \bar{x}_{6} \bar{x}_{5} \bar{x}_{3} \bar{x}_{2} \oplus x_{7} x_{6} x_{5} x_{4} x_{3} x_{2} x_{1} \oplus x_{7} x_{4} \bar{x}_{3} \oplus$ $x_{7} x_{3} \bar{x}_{2} \bar{x}_{1} \oplus x_{7} x_{2} x_{1} \oplus \bar{x}_{6} \bar{x}_{5} x_{3} x_{2} \oplus \bar{x}_{6} \bar{x}_{4} x_{2} \oplus \bar{x}_{6} x_{1} \oplus x_{6} \bar{x}_{5} \bar{x}_{4} \bar{x}_{2} x_{1} \oplus x_{6} x_{5} x_{4} \bar{x}_{2} \oplus x_{6} x_{5} \bar{x}_{3} \oplus$ $\bar{x}_{5} \bar{x}_{4} x_{2} \bar{x}_{1} \oplus x_{5} x_{4} x_{1} \oplus x_{3} x_{2} x_{1}$

$S_{7}^{\{0,1,2,5\}}=\bar{x}_{7} \bar{x}_{6} \bar{x}_{5} \bar{x}_{4} \bar{x}_{3} \bar{x}_{2} \bar{x}_{1} \oplus \bar{x}_{7} \bar{x}_{6} x_{5} x_{4} x_{3} x_{2} x_{1} \oplus \bar{x}_{7} x_{6} x_{5} x_{4} \bar{x}_{3} x_{2} \oplus \bar{x}_{7} x_{6} x_{5} x_{3} \bar{x}_{2} x_{1} \oplus$ $\bar{x}_{7} \bar{x}_{4} \bar{x}_{3} \oplus \bar{x}_{7} x_{2} \bar{x}_{1} \oplus x_{7} x_{6} x_{5} \bar{x}_{4} x_{3} x_{1} \oplus x_{7} x_{6} x_{5} x_{4} x_{2} \bar{x}_{1} \oplus x_{7} x_{6} x_{4} x_{3} x_{2} x_{1} \oplus x_{7} x_{6} \oplus x_{7} x_{5} \oplus$ $x_{7} x_{4} x_{3} \oplus x_{7} \bar{x}_{2} x_{1} \oplus \bar{x}_{6} \bar{x}_{5} \bar{x}_{3} \bar{x}_{2} \oplus \bar{x}_{6} x_{5} \bar{x}_{4} \bar{x}_{3} x_{2} \oplus \bar{x}_{6} x_{5} x_{3} \bar{x}_{2} \bar{x}_{1} \oplus \bar{x}_{6} x_{4} \bar{x}_{1} \oplus x_{6} \bar{x}_{5} x_{3} x_{2} \oplus x_{6} \bar{x}_{4} x_{1} \oplus$ $\bar{x}_{5} \bar{x}_{4} \bar{x}_{2} \oplus \bar{x}_{5} \bar{x}_{3} \bar{x}_{1} \oplus x_{5} \bar{x}_{4} x_{3} x_{2} \bar{x}_{1}$

$S_{7}^{\{0,1,3,4\}}=\bar{x}_{7} \bar{x}_{6} \bar{x}_{5} \bar{x}_{4} \bar{x}_{3} \bar{x}_{2} \oplus \bar{x}_{7} \bar{x}_{6} \bar{x}_{5} x_{4} \bar{x}_{3} x_{2} \bar{x}_{1} \oplus \bar{x}_{7} x_{6} x_{5} \bar{x}_{4} \bar{x}_{3} \bar{x}_{2} \bar{x}_{1} \oplus \bar{x}_{7} x_{6} \oplus \bar{x}_{7} x_{5} \oplus \bar{x}_{7} \bar{x}_{4} x_{3} \oplus$ $\bar{x}_{7} x_{4} \bar{x}_{3} \bar{x}_{2} \bar{x}_{1} \oplus x_{7} \bar{x}_{6} \bar{x}_{5} \bar{x}_{4} x_{3} \bar{x}_{2} \bar{x}_{1} \oplus x_{7} \bar{x}_{6} \bar{x}_{5} \bar{x}_{3} \bar{x}_{1} \oplus x_{7} x_{6} x_{5} x_{4} x_{3} x_{2} x_{1} \oplus x_{7} x_{4} \bar{x}_{3} \oplus x_{7} x_{3} \bar{x}_{2} \bar{x}_{1} \oplus$ $x_{7} x_{2} x_{1} \oplus \bar{x}_{6} \bar{x}_{5} x_{3} x_{1} \oplus \bar{x}_{6} \bar{x}_{4} x_{1} \oplus \bar{x}_{6} x_{2} \oplus x_{6} \bar{x}_{5} \bar{x}_{4} x_{2} \bar{x}_{1} \oplus x_{6} x_{5} x_{4} \bar{x}_{1} \oplus x_{6} x_{5} \bar{x}_{3} \oplus \bar{x}_{5} \bar{x}_{4} \bar{x}_{2} x_{1} \oplus$ $x_{5} x_{4} x_{2} \oplus x_{3} x_{2} x_{1}$

$S_{7}^{\{0,2,3,4,7\}}=\bar{x}_{7} \bar{x}_{6} \bar{x}_{5} \bar{x}_{4} \bar{x}_{1} \oplus \bar{x}_{7} \bar{x}_{6} \bar{x}_{5} \bar{x}_{3} \bar{x}_{2} \oplus \bar{x}_{7} \bar{x}_{6} \bar{x}_{4} x_{3} x_{2} \bar{x}_{1} \oplus \bar{x}_{7} \bar{x}_{6} x_{4} \bar{x}_{3} \bar{x}_{2} x_{1} \oplus$

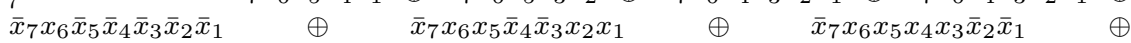
$\bar{x}_{7} x_{6} x_{4} x_{3} x_{2} x_{1} \oplus x_{7} \bar{x}_{6} \bar{x}_{4} \bar{x}_{3} \bar{x}_{2} \bar{x}_{1} \oplus x_{7} x_{6} \bar{x}_{5} \bar{x}_{4} x_{3} \bar{x}_{2} x_{1} \oplus x_{7} x_{6} \bar{x}_{5} x_{4} \bar{x}_{3} x_{2} \bar{x}_{1} \oplus x_{7} x_{6} x_{4} x_{2} \oplus$ $x_{7} x_{6} x_{3} x_{1} \oplus x_{7} \bar{x}_{5} x_{4} x_{3} x_{2} x_{1} \oplus x_{7} x_{5} x_{4} x_{1} \oplus x_{7} x_{5} x_{3} x_{2} \oplus \bar{x}_{6} x_{5} \bar{x}_{4} \bar{x}_{2} \oplus \bar{x}_{6} x_{5} \bar{x}_{3} \bar{x}_{1} \oplus \bar{x}_{6} x_{5} \oplus$ $x_{5} x_{4} x_{3} \oplus x_{5} x_{2} x_{1} \oplus 1$

$23 S_{7}^{\{2,5\}}=\bar{x}_{7} \bar{x}_{6} \bar{x}_{5} x_{4} \bar{x}_{1} \oplus \bar{x}_{7} \bar{x}_{6} x_{5} \bar{x}_{4} \bar{x}_{3} \oplus \bar{x}_{7} x_{6} \bar{x}_{5} \bar{x}_{3} \bar{x}_{2} \oplus \bar{x}_{7} x_{6} x_{5} x_{4} \bar{x}_{3} x_{2} \bar{x}_{1} \oplus$ $\bar{x}_{7} x_{6} x_{5} \bar{x}_{2} \bar{x}_{1} \oplus \bar{x}_{7} x_{6} x_{4} \bar{x}_{3} \bar{x}_{2} x_{1} \oplus \bar{x}_{7} x_{5} x_{3} x_{2} x_{1} \oplus \bar{x}_{7} \bar{x}_{4} x_{3} \bar{x}_{2} \bar{x}_{1} \oplus x_{7} \bar{x}_{6} \bar{x}_{5} x_{1} \oplus x_{7} \bar{x}_{6} x_{4} x_{3} x_{2} \oplus$ $x_{7} x_{6} x_{5} \bar{x}_{4} x_{3} \bar{x}_{2} x_{1} \oplus x_{7} x_{6} x_{3} x_{2} \bar{x}_{1} \oplus x_{7} \bar{x}_{5} \bar{x}_{4} x_{2} \oplus x_{7} x_{5} x_{4} \bar{x}_{3} \bar{x}_{2} \bar{x}_{1} \oplus x_{7} x_{4} x_{3} x_{1} \oplus x_{7} \bar{x}_{3} x_{2} x_{1} \oplus$ $\bar{x}_{6} \bar{x}_{5} x_{4} \bar{x}_{2} \oplus \bar{x}_{6} \bar{x}_{5} x_{3} \oplus \bar{x}_{6} x_{5} \bar{x}_{3} \bar{x}_{2} \bar{x}_{1} \oplus \bar{x}_{6} \bar{x}_{4} x_{2} x_{1} \oplus x_{6} \bar{x}_{5} \bar{x}_{4} \bar{x}_{3} \bar{x}_{1} \oplus x_{6} x_{5} x_{4} \oplus \bar{x}_{5} x_{4} x_{3} x_{2} x_{1}$ $S_{7}^{\{0,2,5\}}=\bar{x}_{7} \bar{x}_{6} x_{5} x_{4} \oplus \bar{x}_{7} \bar{x}_{6} x_{3} x_{2} \oplus \bar{x}_{7} x_{6} \bar{x}_{5} \bar{x}_{3} \bar{x}_{1} \oplus \bar{x}_{7} x_{6} \bar{x}_{4} \bar{x}_{2} \bar{x}_{1} \oplus \bar{x}_{7} \bar{x}_{5} x_{4} x_{3} \bar{x}_{2} x_{1} \oplus$ $\bar{x}_{7} x_{5} \bar{x}_{4} \bar{x}_{3} x_{2} x_{1} \oplus \bar{x}_{7} \oplus x_{7} \bar{x}_{6} x_{5} x_{3} x_{1} \oplus x_{7} \bar{x}_{6} x_{4} x_{2} x_{1} \oplus x_{7} x_{6} \bar{x}_{5} \bar{x}_{2} \oplus x_{7} x_{6} x_{5} x_{4} x_{3} x_{2} x_{1} \oplus$ $x_{7} x_{6} \bar{x}_{4} \bar{x}_{3} \oplus x_{7} \bar{x}_{5} \bar{x}_{4} x_{3} x_{2} \bar{x}_{1} \oplus x_{7} x_{5} x_{4} \bar{x}_{3} \bar{x}_{2} \bar{x}_{1} \oplus \bar{x}_{6} \bar{x}_{5} \bar{x}_{4} \bar{x}_{3} \bar{x}_{2} \oplus \bar{x}_{6} x_{5} x_{2} \bar{x}_{1} \oplus \bar{x}_{6} x_{4} x_{3} \bar{x}_{1} \oplus$ $x_{6} \bar{x}_{5} \bar{x}_{4} x_{1} \oplus x_{6} x_{5} x_{4} x_{3} x_{2} \oplus x_{6} \bar{x}_{3} \bar{x}_{2} x_{1} \oplus \bar{x}_{5} x_{4} \bar{x}_{3} x_{2} \oplus x_{5} \bar{x}_{4} x_{3} \bar{x}_{2} \oplus \bar{x}_{1}$

$S_{7}^{\{0,3,6\}}=\bar{x}_{7} \bar{x}_{6} x_{5} x_{4} x_{3} x_{2} x_{1} \oplus \bar{x}_{7} \bar{x}_{6} \bar{x}_{4} x_{3} \bar{x}_{2} x_{1} \oplus \bar{x}_{7} \bar{x}_{6} x_{4} \bar{x}_{3} x_{2} \bar{x}_{1} \oplus \bar{x}_{7} x_{6} \bar{x}_{5} x_{4} x_{1} \oplus$ $\bar{x}_{7} x_{6} \bar{x}_{5} x_{3} x_{2} \oplus \bar{x}_{7} \bar{x}_{5} \bar{x}_{4} \bar{x}_{3} \bar{x}_{2} \bar{x}_{1} \oplus \bar{x}_{7} x_{5} \bar{x}_{4} \bar{x}_{3} \oplus \bar{x}_{7} x_{5} \bar{x}_{2} \bar{x}_{1} \oplus x_{7} \bar{x}_{6} \bar{x}_{5} x_{4} x_{3} \oplus x_{7} \bar{x}_{6} \bar{x}_{5} x_{2} x_{1} \oplus$ $x_{7} x_{6} x_{5} \bar{x}_{4} \bar{x}_{2} \oplus x_{7} x_{6} x_{5} \bar{x}_{3} \bar{x}_{1} \oplus x_{7} x_{6} \oplus x_{7} \bar{x}_{4} x_{3} x_{2} \bar{x}_{1} \oplus x_{7} x_{4} \bar{x}_{3} \bar{x}_{2} x_{1} \oplus \bar{x}_{6} x_{5} \bar{x}_{4} \bar{x}_{1} \oplus \bar{x}_{6} x_{5} \bar{x}_{3} \bar{x}_{2} \oplus$ $x_{6} \bar{x}_{5} \bar{x}_{4} \bar{x}_{3} \bar{x}_{2} \bar{x}_{1} \oplus x_{6} \bar{x}_{4} \bar{x}_{3} x_{2} x_{1} \oplus x_{6} x_{4} x_{3} \bar{x}_{2} \bar{x}_{1} \oplus \bar{x}_{5} x_{4} x_{2} \oplus \bar{x}_{5} x_{3} x_{1} \oplus x_{5} x_{4} x_{3} x_{2} x_{1}$

$24 S_{7}^{\{0,2,3,5,6\}}=\bar{x}_{7} \bar{x}_{6} \bar{x}_{5} \bar{x}_{3} \bar{x}_{2} x_{1} \oplus \bar{x}_{7} \bar{x}_{6} x_{5} \bar{x}_{4} x_{3} \oplus \bar{x}_{7} \bar{x}_{6} x_{5} x_{4} x_{3} x_{2} x_{1} \oplus \bar{x}_{7} x_{6} \bar{x}_{5} \bar{x}_{4} x_{2} \oplus$ $\bar{x}_{7} x_{6} x_{4} x_{3} x_{2} x_{1} \oplus \bar{x}_{7} x_{6} x_{3} \bar{x}_{2} \bar{x}_{1} \oplus \bar{x}_{7} x_{5} \bar{x}_{3} x_{2} \bar{x}_{1} \oplus \bar{x}_{7} \bar{x}_{4} x_{1} \oplus x_{7} \bar{x}_{6} x_{5} \bar{x}_{4} x_{2} x_{1} \oplus x_{7} \bar{x}_{6} x_{4} \bar{x}_{3} \bar{x}_{1} \oplus$ $x_{7} \bar{x}_{6} \oplus x_{7} x_{6} \bar{x}_{5} \bar{x}_{4} x_{3} x_{1} \oplus x_{7} x_{6} \bar{x}_{5} \oplus x_{7} x_{6} x_{5} x_{4} \bar{x}_{3} \bar{x}_{2} \oplus x_{7} \bar{x}_{5} x_{4} \bar{x}_{2} \bar{x}_{1} \oplus x_{7} \bar{x}_{4} x_{3} x_{2} \oplus \bar{x}_{6} \bar{x}_{5} x_{3} x_{2} \bar{x}_{1} \oplus$ $\bar{x}_{6} x_{4} \bar{x}_{2} \oplus x_{6} x_{5} x_{4} \bar{x}_{1} \oplus x_{6} \bar{x}_{4} \bar{x}_{3} \bar{x}_{2} \bar{x}_{1} \oplus x_{6} \bar{x}_{3} x_{2} x_{1} \oplus \bar{x}_{5} \bar{x}_{4} \bar{x}_{3} \bar{x}_{2} \bar{x}_{1} \oplus \bar{x}_{5} x_{4} \bar{x}_{3} \oplus x_{5} x_{3} \bar{x}_{2} x_{1}$

\section{References}

1. T. Hirayama, G. Koda, Y. Nishitani, and K. Shimizu, Easily testable realization based on single-rail-input OR-AND-EXOR expressions, IEICE Trans. Inf. ES Syst., E82-D (1999) 1278-1286.

2. U. Kalay, D. V. Hall, and M. A. Perkowski, A minimal universal test set for self-test of EXOR-sum-of-products circuits, IEEE Trans. Comput., 49 (2000) 267-276.

3. S. M. Reddy, Easily testable realization for logic functions, IEEE Trans. Comput., 
C-21 (1972) 1183-1188.

4. T. Sasao, Easily testable realizations for generalized Reed-Muller expansions, IEEE Trans. Comput., 46 (1997) 709-716.

5. S. Chattopadhyay, S. Roy, and P. P. Chaudhuri, KGPMIN: an efficient multilevel multioutput AND-OR-XOR minimizer, IEEE Trans. Comput.-Aided Des. Integrated Circuits \& Systems, 16 (1997) 257-265.

6. D. Debnath and T. Sasao, Minimization of AND-OR-EXOR three-level networks with AND gate sharing, IEICE Trans. Inf. ES Syst., E80-D (1997) 1001-1008.

7. R. Ishikawa, T. Hirayama, G. Koda, K. Shimizu, EXOR decomposition with common variables and its application to multiple-output networks, Journal of Circuits, Systems, and Computers, 9 (1999) 83-97.

8. F. Luccio and L. Pagli, On a new boolean function with applications, IEEE Trans. Comput., 48 (1999) 296-310.

9. T. Sasao and P. Besslich, On the complexity of mod-2 sum PLA's, IEEE Trans. Comput., 39 (1990) 262-266.

10. G. Yang, W. N. N. Hung, X. Song, and M. Perkowski, Majority-based reversible logic gates, Theoretical Computer Science, 334 (2005) 259-274.

11. K. Iwama, Y. Kambayashi, and S. Yamashita, Transformation rules for designing CNOT-based quantum circuits, Proc. 39th ACM/IEEE Design Automation Conference, June 2002, pp. 419-424.

12. T. Hirayama, K. Nagasawa, Y. Nishitani, and K. Shimizu, Double fixed-polarity ReedMuller expressions: a new class of AND-EXOR expressions for compact and testable realization, Trans. IPS Japan, 42 (2001) 983-991.

13. T. Sasao, Representations of logic functions using EXOR operators, Representations of Discrete Functions, eds. T. Sasao and M. Fujita (Kluwer Academic Publishers, 1996), pp. 29-54.

14. D. Brand and T. Sasao, Minimization of AND-EXOR expressions using rewrite rules, IEEE Trans. Comput., 42 (1993) 568-576.

15. H. Fleisher, M. Tavel, and J. Yeager, Computer algorithm for minimizing Reed-Muller canonical forms, IEEE Trans. Comput., C-36 (1987) 247-250.

16. M. Helliwell and M. A. Perkowski, A fast algorithm to minimize multi-output mixedpolarity generalized Reed-Muller forms, Proc. 25th ACM/IEEE Design Automation Conference, June 1988, pp. 427-432.

17. Y. Ye and K. Roy, An XOR-based decomposition diagram and its application in synthesis of AND/XOR networks, IEICE Trans. Fundamentals, E80-A (1997) 17421748 .

18. T. Sasao, EXMIN2: A simplification algorithm for exclusive-OR sum-of-products expressions for multiple-valued-input two-valued-output functions, IEEE Trans. Comput.-Aided Des. Integrated Circuits \&5 Systems, 12 (1993) 621-632.

19. N. Song and M. A. Perkowski, Minimization of exclusive sum-of-products expressions for multiple-valued input, incompletely specified functions, IEEE Trans. Comput.Aided Des. Integrated Circuits \& Systems, 15 (1996) 385-395.

20. A. Mishchenko and M. Perkowski, Fast heuristic minimization of exclusive-sums-ofproducts, Proc. 5th Int. Workshop on Reed-Muller, Starkville, Mississippi, USA, August 2001, pp. 242-250.

21. M. A. Perkowski and M. Chrzanowska-Jeske, An exact algorithm to minimize mixedradix exclusive sums of products for incompletely specified Boolean functions, Proc. Int. Symp. Circuits \& Systems, USA, May 1990, pp. 1652-1655.

22. T. Sasao, An exact minimization of AND-EXOR expressions using BDD's, Proc. IFIP WG10.5 Reed-Muller'93, Germany, 1993, pp. 91-98. 
23. S. Stergiou and G. Papakonstantinou, Exact minimization of ESOP expressions with less than eight product terms, Journal of Circuits, Systems, and Computers, 13 (2004) $1-15$.

24. T. Hirayama, Y. Nishitani, and T. Sato, A faster algorithm of minimizing AND-EXOR expressions, IEICE Trans. Fundamentals, E85-A (2002) 2708-2714.

25. Y. Nishitani and K. Shimizu, Lower bounds on size of periodic functions in exclusiveOR sum-of-products expressions, IEICE Trans. Fundamentals, E77-A (1994) 475482.

26. T. Hirayama and Y. Nishitani, A simplification algorithm of AND-EXOR expressions guaranteeing minimality for some subclass of logic functions, IEICE Trans. Inf. E Syst. D-I, J78-D-I (1995) 409-415.

27. N. Koda and T. Sasao, Four variable AND-EXOR minimization expressions and their properties, IEICE Trans. Inf. \& Syst. D-I, J74-D-I (1991) 765-773.

28. N. Koda and T. Sasao, An upper bound on the number of products in minimum ESOPs, Proc. IFIP Workshop Reed-Muller'95, Japan, August 1995, pp. 94-101.

29. N. Koda and T. Sasao, A simplification method for AND-EXOR expressions for multiple-output functions, IEICE Trans. Inf. \& Syst. D-I, J79-D-I (1996) 43-52.

30. T. Hirayama and Y. Nishitani. A simplification algorithm of AND-EXOR expressions for multiple-output functions, Proc. IFIP Workshop Reed-Muller'95, Japan, August 1995 , pp. 88-93.

31. K. McElvain, IWLS'93 Benchmark Set: Version 4.0, Distributed as part of the MCNC International Workshop on Logic Synthesis '93 benchmark distribution, May 1993.

32. T. Sasao, Multiple-valued logic and optimization of programmable logic arrays, IEEE Trans. Comput., 21 (1988) 71-80.

33. S. Even, I. Kohavi, and A. Paz, On minimal modulo-2 sums of products for switching functions, IEEE Trans. Electron. Comput., EC-16 (1967) 671-674.

34. D. Voudouris, M. Sampson, and G. Papakonstantinou, Exact ESCT minimization for functions of up to six input variables, Integr. VLSI J., 41 (2008) 87-105.

35. T. Hirayama and Y. Nishitani, Efficient search methods for obtaining exact minimum AND-EXOR expressions, Proc. 3rd IEEE Int. Workshop on Electronic Design, Test and Applications, Malaysia, January 2006, pp. 137-142.

36. G. Bioul, M. Davio, and J. P. Deschamps, Minimization of ring-sum expansions of Boolean functions, Philips Res. Repts., 28 (1973) 17-36.

37. A. Gaidukov, Algorithm to derive minimum ESOP for 6-variable function, Proc. 5th Int. Workshop on Boolean Problems, September 2002, pp. 141-148.

38. S. Stergiou, D. Voudouris, and G. Papakonstantinou, Multiple-value exclusive-or sumof-products minimization algorithms, IEICE Trans. Fundamentals, E87-A (2004) $1226-1234$.

39. S. Stergiou, K. Daskalakis, and G. Papakonstantinou, A fast and efficient heuristic ESOP minimization algorithm, Proc. 14th ACM Great Lakes symposium on VLSI, April 2004, pp. 78-81. 